\title{
Norsk Epidemiologi
}

\section{Norwegian Journal of Epidemiology}

Årgang 21, supplement 1, november 2011

Utgitt av Norsk forening for epidemiologi

\section{Redaktør:}

Trond Peder Flaten

Institutt for kjemi,

Norges teknisk-naturvitenskapelige

universitet, 7491 Trondheim

Telefon: 73591806

Telefax: 73550877

e-post: trond.flaten@chem.ntnu.no

Abonnement: Tidsskriftet sendes medlemmer av Norsk forening for epidemiologi (NOFE) og abonnerende bibliotek. For å bli medlem eller abonnere, send e-post til NOFE: post@nofe.no. Medlemskontingenten er for tiden kr. 400, det samme er prisen for abonnement. Utenfor Norge er prisen kr. 600.

\section{Adresse NOFE:}

Norsk forening for epidemiologi

KFF v/Olsvik, Paviljongen

Haukeland sykehus

5021 Bergen

Internettadresse for NOFE:

http://www.nofe.no

e-post:post@nofe.no

ISSN 0803-4206

Opplag: 350

Trykk: NTNU-trykk

Layout og typografi: Redaktøren

Tidsskriftet er tilgjengelig online: www.www.ntnu.no/ojs/index.php/norepid Også via Directory of Open Access Journals (www.doaj.org)

Utgis vanligvis med to regulære nummer pr. år. I tillegg kommer supplement med sammendrag fra Norsk forening for epidemiologis årlige konferanse.

\section{DEN NITTENDE NORSKE EPIDEMIOLOGIKONFERANSEN}

\author{
HOLMEN FJORDHOTELL,
} 17.-18. NOVEMBER 2011

VELKOMMEN

PROGRAM

ABSTRACTS

DELTAKERLISTE 


\section{Norwegian Conference of Epidemiology Oslo, 17-18 November 2011}

The 19th conference of the Norwegian Epidemiological association (NOFE) will be held 17-18 November in beautiful surroundings at Holmen Fjordhotell, Oslo. "Assessing causality in theory, practice and health policy" is the main topic of this year's conference. To cover the issue of causality on all three levels we invited three distinguished speakers: Dr. Sonia HernandezDiaz, Associate Professor at Harvard School of Public Health, Andrew David Oxman from the Norwegian Knowledge Centre for the Health Services, and Professor Knut Inge Klepp from the Norwegian Directorate of Health.

Moreover, a special pre-conference course on systematic reviews of epidemiological evidence will be arranged for the participants by the Norwegian Knowledge Centre for the Health Services. NOFE greatly appreciates the Centre's contribution to the conference.

More than 100 participants were registered for this year's conference and we wish all of them a successful conference!

\section{Committee-2011}

Hilde Langseth \& Inger Kristin Larsen (Kreftregistret)

Øyvind Næss \& Andrej Grjibovski (Folkehelseinstituttet) 


\section{Den 19. norske epidemiologikonferansen Holmen fjordhotell 17.-18. november 2011 \\ Oversiktsprogram}

Thursday, November $17^{\text {th }}$

\begin{tabular}{|l|l|}
\hline 10:00-11:00 & Registration / Coffee \\
\hline $11: 00-13: 00$ & $\begin{array}{l}\text { Pre-conference course "Systematic reviews of epidemiological evidence", } \\
\text { part I }\end{array}$ \\
\hline 13:00-14:00 & Lunch \\
\hline 14:00-15:00 & $\begin{array}{l}\text { Pre-conference course “Systematic reviews of epidemiological evidence”, } \\
\text { part II }\end{array}$ \\
\hline 15:00-15:15 & Welcome / Opening \\
\hline 15:15-16:15 & $\begin{array}{l}\text { Guest lecture: “Causal inference and selection bias: The birth weight } \\
\text { paradox". Invited speaker: Dr. Sonia Hernández-Díaz, Harvard School of } \\
\text { Public Health, USA }\end{array}$ \\
\hline $16: 15-16: 30$ & Coffee break \\
\hline $16: 30-17: 30$ & Parallel sessions \\
\hline $17: 30-18: 00$ & “Article of the year" award ceremony \\
\hline 18:00-19:00 & Annual meeting of the Norwegian Epidemiological Society \\
\hline 20:00- & Conference dinner \\
\hline
\end{tabular}

Friday, November $18^{\text {th }}$

\begin{tabular}{|l|l|}
\hline 09:00-10:00 & $\begin{array}{l}\text { Guest lecture: "Systematic reviews: what, why, when, where, who and } \\
\text { how?". Invited speaker: Andrew Oxman, Norwegian Knowledge Centre for } \\
\text { Health Services, Oslo }\end{array}$ \\
\hline $10: 00-11: 30$ & Parallel sessions \\
\hline $11: 30-12: 30$ & Lunch \\
\hline $12: 15-12: 15$ & Parallel sessions \\
\hline & \\
\hline $13: 30-14: 30$ & $\begin{array}{l}\text { Guest lecture: “Causality from the health policy point of view”. Invited } \\
\text { speaker: Prof. Knut Inge Klepp, Norwegian Directorate of Health, Oslo }\end{array}$ \\
\hline $14: 30-15: 30$ & Parallel sessions \\
\hline
\end{tabular}




\section{Den 19. norske epidemiologikonferansen Holmen fjordhotell, 17.-18. november 2010 \\ Detaljert program}

THURSDAY, NOVEMBER $17^{\text {TH }}$

\begin{tabular}{|c|c|}
\hline 10:00-11:00 & Registration / Coffee \\
\hline 11:00-13:00 & $\begin{array}{l}\text { Pre-conference course "Systematic reviews of } \\
\text { epidemiological evidence" }\end{array}$ \\
\hline $13: 00-14: 00$ & Lunch \\
\hline $14: 00-15: 00$ & $\begin{array}{l}\text { Pre-conference course "Systematic reviews of } \\
\text { epidemiological evidence" cont. }\end{array}$ \\
\hline $15: 00-15: 15$ & Welcome / Opening \\
\hline $15: 15-16: 15$ & $\begin{array}{l}\text { Guest lecture: "Causal inference and selection } \\
\text { bias: The birth weight paradox". } \\
\text { Invited speaker: Dr. Sonia Hernández-Díaz, } \\
\text { Harvard School of Public Health, USA }\end{array}$ \\
\hline $16: 15-16: 30$ & Coffee break \\
\hline
\end{tabular}

Parallel sessions A1-A4

Topic: Cancer epidemiology

16:30-16:45 A1 Per Henrik Zahl Spontaneous Regression of Breast Cancers in the Swedish Mammography Screening Program

$16: 45-17: 00$

A2 Signe Opdahl

Placental weight and breast cancer risk in young women

$17: 00-17: 15$

A3 Tom Grotmol

Gene variations in sex hormone pathways and the risk of testicular cancer: A case-parent triad study in a Norwegian-Swedish population

$17: 15-17: 30$

A4 Tron Anders

Eight year costs of treatment for breast cancer Moger in Norway - by TNM stage and detection setting 


\section{Parallel sessions B1-B4}

Topic: Mother and child health

$16: 30-16: 45$

$16: 45-17: 00$

$17: 00-17: 15$

$17: 15-17: 30$
B1 Caroline Fleten

B2 Maria Magnus
Maternal weight change during pregnancy and offspring body mass index at age three years: accounting for shared family factors using sibling analyses

Delivery by Caesarean Section and Early Childhood Respiratory Symptoms and Disorders: the Norwegian Mother and Child Cohort Study

B3 Hedvig Nordeng Fear of childbirth, mental health and medication use during pregnancy

B4 Anna Usynina
Social variations in small-for-gestational-age births in the Murmansk County, Russia: a registry-based study

\section{Parallel sessions C1-C4}

Topic: Infectious diseases epidemiology

$16: 30-16: 45$

$16: 45-17: 00$

$17: 00-17: 15$

$17: 15-17: 30$
C1 Siri Håberg

Pandemic influenza vaccine in pregnancy and fetal death: A study based on nationwide registries in Norway

C2 Irena Jakopanec Trends in HIV infection among men who have sex with men in Norway and coincidental sexually transmitted co-infection, 1995-2009

C3 Olga Ånonsen

EpiNorth: a cooperation project for infectious disease control

C4 Ekaterina Krieger Prevalence of hospital-acquired infections (HAI) in a regional paediatric hospital in Arkhangelsk, Russia in 2010

\section{Plenary}

$17: 30-18: 00$

"Article of the year" award ceremony

18:00-19:00 Annual meeting of the Norwegian Epidemiological Society

\section{Conference dinner}




\section{FRIDAY, NOVEMBER $18^{\text {TH }}$}

\section{Plenary}

09:00-10:00
Guest lecture: "Systematic reviews: what, why, when, where, who and how?". Invited speaker: Andrew Oxman, Norwegian Knowledge Centre for Health Services, Oslo

\section{Parallel sessions A5-A8}

\section{Topic: Lifestyle}

10:00-10:15

$10: 15-10: 30$

$10: 30-10: 45$

$10: 45-11: 00$
A5 Liv Grøtvedt

A6 Randi Selmer

A7 Eivind Ystrøm

A8 Vidar Hjellvik
Does the use of snus in adolescence carry an increased risk of entering adult life as a smoker? A study of Norwegian boys followed from age 16 to 19

Does pattern of maternal use of potentially addictive drugs reproduce in their children?

Is age of alcohol use initiation the cause of alcohol use disorders in young adulthood? A population-based twin study

The relationship between unfavourable lifestyle in the ages 13-25 and subsequent use of potentially addictive prescription drugs in Norwegian men and women

\section{Parallel sessions B5-B9}

Topic: Methods/ causality

10:00-10:15

$10: 15-10: 25$

$10: 25-10: 40$

10:40-10:50

B8 Nathalie Støer

B7 Rani Lill Anjum
Causal inference: Estimation of direct and indirect effects in survival analysis

Analyzing direct and indirect effects of treatment using dynamic path analysis applied to data from the Swiss HIV Cohort Study

The Theory of Causation: Why correlation is not part of causation

Reuse of controls in nested case-control studies with multiple endpoints: A study of prostate cancer and vitamin D

Selection bias: magnitude and limits 


\begin{tabular}{|c|c|c|c|}
\hline \multicolumn{4}{|c|}{$\begin{array}{l}\text { Parallel sessions C5-C8 } \\
\text { Topic: Environmental epidemiology }\end{array}$} \\
\hline \multicolumn{4}{|r|}{$\begin{array}{l}\text { European Study of Cohorts for Air Pollution } \\
\text { Study (ESCAPE) - updates from Oslo, Norway }\end{array}$} \\
\hline $10: 15-10: 30$ & $\mathrm{C} 6$ & Hai-Ying Liu & $\begin{array}{l}\text { ools to facilitate inter-disciplinarity in } \\
\text { nvironmental health decision making }\end{array}$ \\
\hline $10: 30-10: 45$ & $\mathrm{C} 7$ & $\begin{array}{l}\text { Christian } \\
\text { Madsen }\end{array}$ & $\begin{array}{l}\text { The short-term effect of } 24 \text {-hour average and } \\
\text { peak air pollution on mortality in Oslo, Norway }\end{array}$ \\
\hline $10: 45-11: 00$ & & Cecilie Dahl & $\begin{array}{l}\text { Is the quality of drinking water a risk factor for } \\
\text { forearm fractures? Cohort of Norway }\end{array}$ \\
\hline
\end{tabular}

\section{Parallel sessions A9-A13}

Topic: Environmental epidemiology

$\begin{array}{llll}\text { 11:00-11:15 } & \text { A9 } & \begin{array}{l}\text { Christian } \\ \text { Madsen }\end{array} & \begin{array}{l}\text { Comparison of land-use regression models for } \\ \text { predicting spatial } \mathrm{NO}_{\mathrm{x}} \text { contrasts over a three } \\ \text { year period in Oslo, Norway }\end{array} \\ \text { 11:15-11:30 } & \text { A10 } & \begin{array}{l}\text { Sviatlana } \\ \text { Panasevich }\end{array} & \begin{array}{l}\text { Road traffic noise, air pollution and hyper- } \\ \text { tension in a population-based sample in Oslo }\end{array} \\ \text { 11:30-12:30 } & & \text { Lunch } \\ \text { 12:30-12:45 } & \text { A11 } & \text { Geir Aamodt } & \text { Water supply and prevalence of ulcerative } \\ & & & \begin{array}{l}\text { Colitis using Cohort of Norway and the } \\ \text { Norwegian waterworks registry }\end{array} \\ \text { 12:45-13:00 } & \text { A12 } & \text { Kristin Holvik } & \begin{array}{l}\text { Hip fracture incidence in Norway 1999-2008: } \\ \text { Norwegian Epidemiologic Osteoporosis Studies }\end{array}\end{array}$

\section{Parallel sessions B10-B14 \\ Various topics}

\begin{tabular}{|c|c|c|c|}
\hline 11:00-11:15 & B10 & $\begin{array}{l}\text { Anne Johanne } \\
\text { Søgaard }\end{array}$ & $\begin{array}{l}\text { Weight cycling increase the risk of forearm } \\
\text { fractures in men. The Troms } \varnothing \text { Study }\end{array}$ \\
\hline $11: 15-11: 30$ & B11 & Anna Biehl & $\begin{array}{l}\text { Does instrument error impact the estimated } \\
\text { prevalence of overweight and obesity in } \\
\text { population-based surveys? }\end{array}$ \\
\hline $11: 30-12: 30$ & & & Lunch \\
\hline $12: 30-12: 45$ & B12 & $\begin{array}{l}\text { Svetlana } \\
\text { Skurtveit }\end{array}$ & $\begin{array}{l}\text { Agreement between self-reported data on } \\
\text { medicine use in the Norwegian Mother and } \\
\text { Child cohort study and prescription records }\end{array}$ \\
\hline $12: 45-13: 00$ & B13 & Eivind Bjerkaas & $\begin{array}{l}\text { Smoking prevalence in the "Smoking, Women } \\
\text { and Cancer Study" compared to smoking } \\
\text { prevalence in the official Norwegian statistics }\end{array}$ \\
\hline
\end{tabular}




\section{Parallel sessions C9-C13}

Topic: Paediatric epidemiology

11:00-11:15

$11: 15-11: 30$

$11: 30-12: 30$

$12: 30-12: 45$

$12: 45-13: 00$
C9 Kari Kveim Lie

C10 Ragnhild Hovengen

C11 Bror Just Andersen

C12 Elisabet Wits $\varnothing$
Register prevalence of autism spectrum disorders, ADHD, epilepsy and cerebral palsy in Norwegian children

The Norwegian Child Growth Study: Trends in height, weight and waist circumference among third graders (8-9 yrs), 2008 - 2010

\section{Lunch}

Effects of preventive mental health programs in schools. A longitudinal quasi experimental intervention study with Solomons design

Polymorphisms in the innate immune IFIH1 gene, frequency of enterovirus in monthly fecal samples during infancy, and islet autoimmunity

\section{Plenary}

13:00-14:00

Guest lecture: "Causality from the health policy point of view". Invited speaker: Prof. Knut Inge Klepp, Norwegian Directorate of Health, Oslo

\section{Parallel sessions A13-A16}

Various topics
14:00-14:15
A13 Lars Christian Stene
Enterovirus and Type 1 Diabetes: review and meta-analysis of observational studies
$14: 15-14: 30$
A14 Børge Moe
The combined effect of leisure time physical activity and diabetes on cardiovascular mortality: the HUNT Study, Norway
$14: 30-14: 45$
A15 Kristi Lund Vik
Generational studies of cardiovascular risk factors in Nord-Trøndelag: The HUNT Study 


\section{Parallel sessions B14-B17}

\section{Various topics}

14:00-14:15 B14 Karina Olsen The balance linoleic acid and alpha-linolenic acid in the blood affects inflammatory blood gene expression profiles

$14: 15-14: 30$

B15 Karina Olsen

Vitamin D and Staphylococcus aureus carriage The Troms $\varnothing$ Staph and Skin Study

$14: 30-14: 45$

B16 Ingvild

Maternal serum levels of 25-hydroxy-vitamin D Sørensen during pregnancy and risk of type 1 diabetes in the offspring

$14: 45-15: 00$

B17 Alexander Epidemiology of pedestrian traffic accidents in Kudryavtsev Arkhangelsk, Russia, 2005-2010

\section{Parallel sessions C14-C17}

Topic: Cancer/Methods

\begin{tabular}{|c|c|c|c|}
\hline $14: 00-14: 15$ & C13 & Asbjørn Westvik & $\begin{array}{l}\text { A statistical simulation model of the natural } \\
\text { history of breast cancer }\end{array}$ \\
\hline $14: 15-14: 30$ & C14 & Morten Valberg & $\begin{array}{l}\text { Frailty modeling of age-incidence of } \\
\text { osteosarcoma and Ewing sarcoma for } \\
\text { individuals below } 40 \text { years }\end{array}$ \\
\hline $14: 30-14: 45$ & C15 & Ranjan Parajuli & $\begin{array}{l}\text { Colorectal cancer incidence in "Smoking, } \\
\text { Women and Cancer Study" compared with the } \\
\text { Norwegian general female population }\end{array}$ \\
\hline $14: 45-15: 00$ & C16 & $\begin{array}{l}\text { Harald } \\
\text { Weedon-Fekjær }\end{array}$ & Understanding recent breast cancer trends \\
\hline
\end{tabular}




\title{
A1
}

\section{Spontaneous Regression of Breast Cancers in the Swedish Mammography Screening Program}

\author{
Per-Henrik Zahl, ${ }^{1}$ Peter C. Gøtzsche², Jan Mæhlen ${ }^{3}$ \\ ${ }^{1}$ Norwegian Institute of Public Health, Norway \\ 2 Nordic Cochrane Centre, Denmark \\ ${ }^{3}$ Department of Pathology, Oslo University Hospital, Norway
}

Introduction: Mammography screening has been associated with a persistent $50 \%$ breast cancer incidence increase. An analysis of the incidence change during the introduction of the Norwegian screening program in the late 1990's suggested that the natural history of many screen-detected invasive breast cancers is to regress spontaneously, but the study was criticized for possible confounding by use of hormone replacement therapy.

Aims: To study regression of breast cancer in a population where women did not use hormone replacement therapy.

Methods: We compared cumulative breast cancer incidence in age-matched cohorts of women residing in 7 Swedish counties before and after the initiation of public mammography screening in the period 1986-1990. A screened group including all women aged 40-69 years $(n=328,927)$ was followed for 6 years after the first invitation to the program. A staggered control group including all women in the same age range 40-69 years $(n=317,404)$, was also followed for 6 years; 4 years without screening and 2 years when they entered the screening program. Screening attendance was $80 \%$ in both groups. Data were obtained from the Swedish Cancer and the Causes-of-Death Registry. Our method adjusts for lead-time and length time bias and can be used to study changes in breast cancer mortality too.

Results: Compared to the control group, the screened group had 14\% higher 6-year cumulative incidence of invasive breast cancer (1443 vs. 1269 per 100,000 population; relative risk, 1.14; 95\% CI, 1.10-1.18). The absolute difference in cumulative incidence rates between the test and control group persisted at 10 years extended follow-up, and women invited to screening had 35\% more breast cancers (35\% overdiagnosis). Almost all differences in breast cancer mortality disappeared after adjusting for improved treatment.

Conclusions: Almost all overdiagnosed cancers must regress and there was no reduction in the breast cancer mortality when screening with mammography. 


\section{A2}

\section{Placental weight and breast cancer risk in young women}

Signe Opdahl' ${ }^{1}$, Mirjam DK Alsaker ${ }^{1,2}$, Pål R Romundstad ${ }^{1}$, Anne Eskild ${ }^{3,4}$ and Lars J Vatten ${ }^{1}$

1 Department of Public Health and Community Medicine, Norwegian University of Science and Technology, N-7491 Trondheim, Norway

2 Department of Oncology, St. Olavs Hospital, N-7006 Trondheim, Norway

3 Department of Obstetrics and Gynecology, Akershus University Hospital and the Faculty of Medicine, University of Oslo, Norway

4 Divisions of Epidemiology and Mental Health, Norwegian Institute of Public Health, Oslo, Norway

Introduction: Pregnancy has a short term risk increasing effect on breast cancer that may be attributed to growth promoting effects of pregnancy hormones on prevalent but undetected breast tumours. Results of two previous studies suggested that placental weight, a possible indicator of pregnancy hormone levels, may be associated with maternal breast cancer risk.

Aims: To assess whether a high placental weight is associated with increased breast cancer risk.

Methods: In a cohort of 338051 women followed from 1999 to 2008, based on data linkage between the Medical Birth Registry and the Cancer Registry of Norway, we assessed whether placental weight in a woman's most recent pregnancy was related to breast cancer risk during the first years following pregnancy. Cox proportional hazards models were used to estimate hazard ratios with 95\% confidence intervals, treating placental weight as a time dependent exposure.

Results: During follow-up (median 6.0 years, interquartile range 3.0-8.3 years), 648 women were diagnosed with breast cancer at a mean age of 38.4 years (standard deviation 5.3 years). Placental weight in the most recent pregnancy was not associated with breast cancer risk: the hazard ratio per 100 gram increase in placental weight was 1.03 (95\% confidence interval $0.96,1.10$ ). There was a similar lack of association related to mean placental weight across pregnancies, and to placental weight associated with the first birth.

Conclusion: We could not confirm previous reports that women who develop large placentas are at increased risk of breast cancer. More research is needed to identify factors that influence breast cancer risk in young women. 


\title{
A3
}

\section{Gene variations in sex hormone pathways and the risk of testicular cancer: A case-parent triad study in a Norwegian-Swedish population}

\author{
Kristiansen $W^{1}$, Andreassen $\mathrm{KE}^{2}$, Karlsson R ${ }^{3}$, Aschim EL ${ }^{1}$, Bremnes RM ${ }^{4}$, Dahl 0 ${ }^{5}$, Fosså \\ $\mathrm{SD}^{6,7}$, Klepp $\mathrm{O}^{8}$, Langberg $\mathrm{CW}^{9}$, Solberg $\mathrm{A}^{10}$, Tretli $\mathrm{S}^{2}$, Adami $\mathrm{H}-\mathrm{O}^{3,11}$, Wiklund $\mathrm{F}^{3}$, Haugen \\ $\mathrm{TB}^{1}$, Grotmol T ${ }^{2}$ \\ ${ }^{1}$ Faculty of Health Sciences, Oslo and Akershus University College of Applied Sciences, Oslo, Norway \\ ${ }^{2}$ Dept of Etiologic Research, Cancer Registry of Norway, Oslo, Norway \\ ${ }^{3}$ Dept of Medical Epidemiology and Biostatistics, Karolinska Institutet, Stockholm, Sweden \\ ${ }^{4}$ Translational Cancer Research Group, Institute of Clinical Medicine, University of Tromsø and Dept of \\ Oncology, University Hospital North Norway, Troms $\emptyset$, Norway \\ ${ }^{5}$ Section of Oncology, Institute of Medicine, University of Bergen and Dept of Oncology, Institute of \\ Medicine, Haukeland University Hospital, Bergen, Norway \\ ${ }^{6}$ Dept of Clinical Cancer Research, The Norwegian Radium Hospital, Oslo University Hospital, Oslo, Norway \\ ${ }^{7}$ Faculty of Medicine, The Norwegian Radium Hospital, University of Oslo, Oslo, Norway \\ ${ }^{8}$ Dept of Oncology, Ålesund Hospital, Helse Sunnmøre HF, Ålesund, Norway \\ ${ }^{9}$ Cancer Centre, Ullevål University Hospital, Oslo, Norway \\ ${ }^{10}$ Dept of Oncology, St Olavs University Hospital, Trondheim, Norway \\ ${ }^{11}$ Dept of Epidemiology, Harvard School of Public Health, Boston, MA, USA
}

Introduction: Testicular cancer (TC) is the most common cancer in young men, and an imbalance between the oestrogen and androgen levels in utero is hypothesized to influence TC risk. Thus, polymorphisms in genes involved in the action of sex hormones may contribute to variability in an individual's susceptibility to TC.

Aims: The first aim was to examine if there is any association between polymorphisms in sex hormone genes and TC risk. Further aims were to investigate whether the allelic effect on TC risk was modified by histological subgroup, by country, or the gender of the parent from whom the allele was transmitted.

Methods: We conducted a large Norwegian-Swedish population-based case-parent triad study, genotyping 105 SNPs in 20 sex hormone pathway genes in 831 complete triads and 474 dyads. To increase statistical power, the analysis was expanded to include 712 case singletons and 3922 controls from the TwinGene project, thus including triads, dyads, and the case-control sample in a single test for association.

Results: We found three htSNPs capturing the common genetic variation across the ESR2 gene (encoding ER $\beta$ ) statistically significantly associated with the risk of TC. The G allele of the rs12435857 SNP was associated with increased risk of TC (OR=1.15, 95\% CI=1.07$\left.1.24, P_{\text {adj }}=0.026\right)$, while the $\mathrm{T}$ alleles of the rs $12434245 \mathrm{SNP}(\mathrm{OR}=0.66,95 \% \mathrm{CI}=0.53-0.82$, $\left.P_{\text {adj }}=0.018\right)$ and the $r s 10137185$ SNP (OR=0.79, 95\% CI=0.70-0.90, $\left.P_{\text {adj }}=0.031\right)$ were associated with reduced risk of TC after Bonferroni correction. No statistically significant differences in allelic effect estimates were found between the histologic subtypes, the Norwegian and the Swedish populations, or between maternally and paternally inherited genetic variation in sex hormone pathways and TC risk.

Conclusion: Our findings provide supportive evidence for ER $\beta$ being implicated in the aetiology of TC. Exploring the functional role of the TC-risk associated htSNPs will further elucidate the biological mechanisms involved. 


\title{
A4
}

\section{Eight year costs of treatment for breast cancer in Norway - by TNM stage and detection setting}

\author{
Gudrun Maria Waaler Bjørnelv ${ }^{1}$, Eline Aas ${ }^{12}$, Ivar Sønbø Kristiansen ${ }^{1}$ and \\ Tron Anders Moger ${ }^{13}$ \\ 1. University of Oslo, Institute of health and society, Department of Health Economics and Health \\ Management \\ 2. Health Economics Research Program at the University of Oslo (HERO) \\ 3. University of Oslo, Institute of basic medical sciences, Department of biostatistics
}

Introduction: If mammography screening leads to that breast cancer is discovered at an earlier stage, less extensive initial treatment and lower treatment costs may be expected. On the contrary, if screening prolongs life expectancy and thus prolongs the period when the patient needs treatment, higher treatment costs may occur.

Aims: The purpose of this study was to calculate treatment costs for breast cancer,
exploring differences between screening status within TNM stages at diagnosis.

Methods: All women in screening ages in Norway, between 50 and 69 years, who were been diagnosed with breast cancer between January $1^{\text {st }} 2001$ and December $31^{\text {st }} 2008$ were included for analyses. Actual resource use in hospitals in the period January $1^{\text {st }}$ 2008 thru April 30th 2009 was collected from the Norwegian Patient Registry (NPR). Expected treatment costs due to breast cancer was calculated as a function of the mean cost and the survival probability $t$ months after diagnosis by stage at diagnosis and detection setting.

Results: Costs in the first eight years after diagnosis for TNM stage I cancers that were detected in screening and outside of screening, respectively, were $\mathrm{kr} 2.1 \mathrm{mil}$. and $4.0 \mathrm{mil}$. For stage II, III and IV, treatment costs for cancers detected in screening and outside of screening the first eight years were 4.8 mil. and 5.8 mil. (stage II), 4.3 mil. and 6.7 mil. (stage III), and 6.5 mil. and 6.2 mil. (stage IV), respectively.

Conclusions: The preliminary results show that treatment costs tend to differ for similarly staged cancers in different screen settings. Thus, screening might lead to detection of cancers, not only in earlier stages, but also with lower severity within stages. This might also be due to a better clinical pathway for cancers detected in a screening setting, due to well organized mammography program. 
B1

\section{Maternal weight change during pregnancy and offspring body mass index at age three years: accounting for shared family factors using sibling analyses}

Caroline Fleten 1 , Hein Stigum¹, Wenche Nystad1, Rolv Skjærven²,3, Debbie A. Lawlor ${ }^{4}$, George Davey Smith ${ }^{4}$, Øyvind Næss ${ }^{1}$

${ }^{1}$ Division of Epidemiology, Norwegian Institute of Public Health, Oslo, Norway

2 University of Bergen, Department of Public Health and Primary Health Care, Bergen, Norway

3 Medical Birth Registry of Norway, Norwegian Institute of Public Health, Bergen, Norway

${ }^{4}$ MRC Centre for Causal Analyses in Translational Epidemiology, School of Social and Community Medicine, University of Bristol, Bristol, England

Introduction: Previous studies have shown associations between increasing maternal weight gain during pregnancy and offspring adiposity during childhood. However, the findings may be confounded by unmeasured genetic or environmental obesogenic factors shared by siblings.

Aim: To investigate the association between maternal weight change during pregnancy and offspring body mass index (BMI) at age three years using sibling analyses.

Material and methods: Mothers and offspring were recruited during pregnancy in 20012007 in the Norwegian Mother and Child Cohort Study (MoBa) conducted by The Norwegian Institute of Public Health. Data from self-administered questionnaires were used in linear multilevel regression analyses.

Preliminary results: The analyses consist of 4,064 offspring with $\geq 1$ siblings in MoBa. Mean offspring BMI was $16.1 \mathrm{~kg} / \mathrm{m}^{2}$ (SD 1.5) and mean maternal weight change during pregnancy was $14.6 \mathrm{~kg}$ (SD 5.4). The crude between-mothers effect estimate showed $0.016 \mathrm{~kg} / \mathrm{m}^{2}$ (95\% CI: 0.006, 0.027; $\mathrm{P}=0.003$ ) higher offspring BMI per $1 \mathrm{~kg}$ increment in maternal weight change, and the crude within-mothers effect estimate showed 0.002 $\mathrm{kg} / \mathrm{m}^{2}$ (95\% CI: $-0.017,0.012 ; \mathrm{P}=0.744$ ) lower offspring BMI per $1 \mathrm{~kg}$ increment in maternal weight change. After adjustments, these estimates were $0.021 \mathrm{~kg} / \mathrm{m}^{2}$ (95\% CI: 0.010 , 0.032; $\mathrm{P}<0.001)$ and $0.005 \mathrm{~kg} / \mathrm{m}^{2}(95 \% \mathrm{CI}:-0.011,0.020 ; \mathrm{P}=0.543)$ higher offspring $\mathrm{BMI}$, respectively.

Conclusion: This population-based study found that greater maternal weight gain during pregnancy was associated with higher offspring BMI at age three years, but that this association may be explained by shared familial factors, such as genes and environment. 


\title{
B2
}

\section{Delivery by caesarean section and early childhood respiratory symp- toms and disorders: the Norwegian Mother and Child Cohort Study}

\author{
Maria C. Magnus (1), Siri E. Håberg (1), Hein Stigum (1,4), Per Nafstad (1,4), Stephanie J. \\ London (3), Siri Vangen (1,2) and Wenche Nystad (1) \\ 1. Division of Epidemiology, Norwegian Institute of Public Health, Oslo, Norway \\ 2. Women and Children's division, Oslo University Hospital, Rikshospitalet, Oslo, Norway \\ 3. Epidemiology branch and Laboratory of Respiratory Biology, National Institute of Environmental Health \\ Sciences, National Institutes of Health, Department of Health and Human Services, Research Triangle \\ Park, North Carolina \\ 4. Department of General Practice and Community Medicine, Medical Faculty, University of Oslo, Norway
}

Introduction: Studies have indicated that children delivered by caesarean section are at an increased risk of wheezing and asthma. This may be the result of an altered immune system development, due to a delayed gut colonization, or increased neonatal respiratory morbidity.

Aims: The current study examined the associations between delivery by caesarean section and development of wheezing, asthma and recurrent lower respiratory tract infections (LRTI) up to 36 months of age.

Methods: The study population consisted of 37,171 children in the Norwegian Mother and Child Cohort Study (MoBa). This sample included children of mothers who had completed the questionnaires administered at 18 and 30 weeks gestation, in addition to the questionnaires when the child was 6,18 and 36 months old. Data from version V of MoBa was analyzed. Generalized Linear Models were used in the multivariable analysis. Multiple imputation techniques were used to examine the influence of missing information on the associations using data augmentation, a Markov Chain Monte Carlo method.

Results: Children delivered by caesarean section had an increased likelihood of current asthma at 36 months, RR 1.17 [95\% CI: 1.03, 1.32], where the association was stronger amongst children of non-atopic mothers, RR 1.33 [95\% CI: 1.12, 1.58]. The association between delivery by caesarean section and asthma was found to not go through duration of breastfeeding or day care attendance, by using inverse probability weighting to control for these two intermediate factors. No increased risk of wheezing or recurrent LRTI was seen amongst children delivered by caesarean section. Findings were similar amongst those delivered by acute and elective caesarean section.

Conclusions: Children delivered by caesarean section may have an increased risk of current asthma at 36 months, but residual confounding cannot be excluded. Future prospective studies should re-examine this association in different age groups.

Publication: American Journal of Epidemiology (In press) 


\title{
B3
}

\section{Fear of childbirth, mental health and medication use during pregnancy}

\author{
Hedvig Nordeng 1, 2, Cathrine Hansen ${ }^{1}$, Susan Garthus-Niegel2, 3, Malin Eberhard-Gran ${ }^{2,3}$ \\ ${ }^{1}$ Department of Pharmacy, School of Pharmacy, University of Oslo, Oslo, Norway \\ ${ }^{2}$ The Division for Mental Health, National Institute of Public Health, Oslo, Norway \\ ${ }^{3}$ Health Services Research Centre, Akershus University Hospital, Norway
}

Introduction: Approximately $20 \%$ of all pregnant women fear giving birth. The number of planned caesarean sections performed due to childbirth-related anxiety has increased markedly in recent years. Hence, fear of childbirth is an important women's health issue. No prior studies have investigated whether there is a relationship between fear of childbirth and medication use during pregnancy.

Aims: To investigate whether medication use during pregnancy is associated with fear of childbirth and maternal mental health. Psychotropics and analgesics were specifically studied.

Methods: The study is a cohort study including pregnant women at Akershus University Hospital, Norway between November 2008 and April 2010. Data were collected by three self completed questionnaires at pregnancy week 17 and 32 and 8 weeks post partum. Fear of childbirth was measured by the Wijma Delivery Expectancy Questionnaire (WDEQ). Symptoms of anxiety were measured by the Hopkins Symptom Check List (SCL-25) and symptoms of depression by the Edinburgh Postnatal Depression Scale (EPDS).

Results: We included 1984 women who had delivered by November 2010 and who had filled out all three questionnaires (59\%). Analgesics use was reported by $55.8 \%$ of the women and psychotropic medication use by $1.8 \%$. In all, $7.8 \%$ of the women reported fear of childbirth, $11.8 \%$ reported symptoms of anxiety and $8.1 \%$ reported symptoms of depression. Fear of childbirth was significantly associated with use of psychotropic drugs (OR 3.63, 95\% CI 1.39-9.43), but not use of analgesics or medications in general. The presence of anxiety or depression increased the magnitude of this association.

Conclusion: Fear of childbirth is associated with an increased use of psychotropic medication. This finding could not only explained by an overlap between fear of childbirth and mental disorders. In order to improve the clinical approach to women who fear childbirth, better understanding of such fear is necessary. 


\title{
B4
}

\section{Social variations in small-for-gestational-age births in the Murmansk County, Russia: a registry-based study}

\author{
Anna Usynina ${ }^{1,2}$, Andrej M Grjibovski ${ }^{1,3,4}$ \\ 1. International School of Public Health, Northern State Medical University, Arkhangelsk, Russia \\ 2. Department of Neonatology and Perinatology, Northern State Medical University, Arkhangelsk, Russia \\ 3. Norwegian Institute of Public Health, Oslo, Norway \\ 4. Institute of Community Medicine, University of Troms $\emptyset$, Norway
}

Introduction: Small-for-gestational-age (SGA) infants are at increased risk of morbidity and mortality in infancy and childhood and represent an important public health issue, particularly in low- and middle-income countries. Social variations in the prevalence of SGA have been extensively studied in many countries, but the evidence from Russia is very scarce.

Aims: To investigate the associations between SGA and selected socio-demographic characteristics using the data from the Murmansk County Birth Registry (MCBR).

Methods: Data on still- or live-born singleton infants at gestational age of 34-41 weeks born in 2006-2008 in the Murmansk region were obtained from the MCBR $(n=21,499)$. Binary logistic regression was used to assess associations between SGA (birth weight $<10^{\text {th }}$ percentile for given gestational age) and maternal education, occupation, marital status with adjustment for confounders such as parity, age, maternal smoking and alcohol consumption.

Results: The prevalence of SGA by maternal education varied from $20.7 \%$ in mothers with basic education vs. $7.1 \%$ in women with higher education. After adjustment for other studied variables women with basic education were twice as likely to deliver SGA babies as women with higher education (OR=2.12, 95\% CI: 1.65, 2.72). Unemployed $(\mathrm{OR}=1.25,95 \% \mathrm{CI}: 1.06-1.46)$, cohabiting $\mathrm{OR}=1.40,95 \% \mathrm{CI}: 1.25-1.57$ and single $(\mathrm{OR}=1.26$, 95\%CI: 1.09-1.44) women were also more likely to deliver SGA babies.

Conclusions: Social variations in SGA births in the Murmansk county of Russia are most pronounced by maternal education similarly to variation in other pregnancy outcomes in other Russian regions. Further research should address the factors behind these variations. 


\section{C1}

\section{Pandemic influenza vaccine in pregnancy and fetal death: A study based on nationwide registries in Norway}

Siri E Håberg1, Lill Trogstad ${ }^{1}$, Nina Gunnes ${ }^{1}$, Håkon Gjessing ${ }^{1}$, Anders Skrondal' ${ }^{1}$, Sven Ove Samuelsen ${ }^{1}$, Inger Cappelen ${ }^{1}$, Anders Engeland ${ }^{1}$ Preben Aavitsland ${ }^{1}$, Steinar Madsen ${ }^{2}$, Ingebjørg Buajordet ${ }^{2}$, Kari Furu ${ }^{1}$, Per Nafstad ${ }^{1}$, Stein Emil Vollset ${ }^{1}$, Berit Feiring ${ }^{1}$, Hanne Nøkleby ${ }^{1}$, Per Magnus ${ }^{1}$, Camilla Stoltenberg ${ }^{1}$

1 Norwegian Institute of Public Health, Norway

2 Norwegian Medicines Agency, Oslo, Norway

Introduction: During the H1N1 pandemic, vaccination was recommended for pregnant women. Reports of miscarriages and fetal death after vaccination raised concerns about the safety of the adjuvanted influenza vaccine used in Norway.

Aim: The aim of this study was to estimate the association between pandemic vaccination during pregnancy and fetal death.

Methods: Four population based registries were linked to provide vaccination status, birth records and background information on all women in Norway who gave births from January $1^{\text {st }} 2009$ through October $14^{\text {th }} 2010$. We used Cox regression with time to fetal death (in gestational days) as survival time to estimate crude and adjusted hazard ratios (HR) for fetal death after vaccination with adjuvant influenza vaccines. Vaccination status and exposure to the pandemic peak during pregnancy were used as time-dependent covariates.

Results: Vaccinated women had a lower risk of fetal death compared to unvaccinated women.

Conclusion: Vaccination with adjuvanted pandemic vaccine during pregnancy was associated with a decreased risk of fetal death. 


\title{
C2
}

\section{Trends in HIV infection among men who have sex with men in Norway and coincidental sexually transmitted co-infection, 1995-2009}

\author{
Irena Jakopnec ${ }^{1,2}$, Andrej M. Grjibovski1 ${ }^{1,3}, \emptyset_{\text {ivind Nilsen }}{ }^{1}$, Hans Blystad ${ }^{1}$, Preben Aavitsland ${ }^{1}$ \\ ${ }^{1}$ Norwegian Institute of Public Health, Oslo, Norway \\ 2 Det Norske Veritas, Oslo, Norway \\ ${ }^{3}$ International School of Public Health, Northern State Medical University, Arkhangelsk, Russia
}

Introduction: In 2004, an outbreak of HIV was reported among MSM in Norway. This was accompanied by a simultaneous increase of syphilis and gonorrhoea. Among syphilis MSM cases, as many as $39 \%$ were reported to be co-infected with HIV in 2008. Coexisting HIV and other sexually transmitted infections (STI) can mutually enhance their transmission.

\begin{abstract}
Aims: We aimed to describe socio-demographic, behavioural and other characteristics of newly reported HIV positive men who have sex with men (MSM) in Norway from 1995 to 2009 in order to learn about the prevalence of co-infections among HIV positive and to guide preventive measures.
\end{abstract}

Methods: We collected the data from the Norwegian Surveillance System for Communicable Diseases and described the cases by age, clinical presentation of HIV infection, previous HIV test, STI co-infection and source partner. We used simple linear regression to estimate trends over time.

Results: In the study period, 807 MSM, aged from 17 to 80 years, were newly diagnosed with HIV. Upon diagnosis, 59\% (479) was asymptomatic and a third (265, 33\%) had never been tested before. STI co-infection was reported in $98(12 \%)$. We observed an increase in: total number of HIV positive MSM, clinically acute HIV infections, STI coinfections, including syphilis and the proportion of those being infected by a casual partner (in all $\mathrm{p}$ for trend $=0.001$ or less).

Conclusions: The HIV epidemic among MSM in Norway continues. It is likely fuelled by an increasing prevalence of risky sex with casual partners and by increasing number of HIV positives with an STI co-infection. Sexually active MSM should be regularly tested for HIV and STI and, if found positive, a thorough contact tracing should be conducted. 


\title{
C3
}

\section{EpiNorth: a cooperation project for infectious disease control}

\author{
Olga Ånonsen, Bjørn G. Iversen \\ Norwegian Institute of Public Health
}

Introduction: The project was initiated in 1999 to establish and maintain an effective network for disease prevention and control. Collaborating partners are the public health institutes in Nordic and Baltic countries, Poland, Ukraine, Belarus and 11 regions in Northwestern Russia. The official languages of the project are English and Russian. Ministry of Health and Care Services of Norway, European Center for Disease Control and participating institutes are the main contributors.

\begin{abstract}
Aim: The aim of the EpiNorth project is to improve communicable diseases control, prevention and communication across borders and language barriers.

Methods: The EpiNorth website consists of several modules containing updated information on infectious diseases in the region and beyond. The EpiNorth Journal publishes articles from EpiNorth's community. EpiTrain courses gather junior and experienced specialists in epidemiology and give opportunity exchange knowledge and strengthen networks.
\end{abstract}

Results: EpiNorth website launched in 2004 provides nearly daily updated information on epidemic intelligence in the region (EpiWatch), a database of teaching materials updated after every EpiTrain course, and a database containing surveillance data on 44 infectious diseases since 1999 from all participating countries (Russian territories provide separate data). EpiNorth Journal publishes 4 issues a year and is distributed to about 3500 subscribers in 45 countries. Main topics are surveillance reports and outbreak investigations. EpiNorth also collaborates with other networks, like the European Programme for Intervention Epidemiology Training, the European surveillance network for selected vaccine-preventable diseases, and the Network for Communicable Disease Control in Southern Europe and Mediterranean Countries. Nowadays, the main objectives are increased focus on epidemic intelligence and continued bridging through common activities.

Conclusion: The EpiNorth project has become an inevitable part of collaboration between public health institutes and has created a wide network of epidemiologists and other specialists working with infectious diseases on national and local levels in respective countries. 


\title{
C4
}

\section{Prevalence of hospital-acquired infections (HAI) in a regional paediatric hospital in Arkhangelsk, Russia in 2010}

\author{
Ekaterina Krieger ${ }^{1-2}$, Olga Samodova ${ }^{2}$, Hanne Merete Eriksen ${ }^{3}$, Andrej M Grjibovski ${ }^{1,3,4}$ \\ 1. International School of Public Health, Northern State Medical University, Arkhangelsk, Russia \\ 2. Department of Communicable Diseases, Northern State Medical University, Arkhangelsk, Russia \\ 3. Department of Infectious Disease Epidemiology, Norwegian Institute of Public Health, Oslo, Norway \\ 4. Institute of Community Medicine, University of Tromsø, Norway
}

Introduction: Surveillance of hospital-acquired infections (HAI) in Russia is considered to suffer from underreporting and use of questionable criteria. The first survey on the prevalence HAIs in Northwest Russia was performed in the Arkhangelsk regional paediatric hospital in cooperation with Norwegian Institute of Public Health in 2006 and revealed a prevalence of $\mathrm{HAI}$ of $17 \%$.

Aims: The aim of this study was assess the prevalence of HAI and their correlates in the same hospital 5 years after establishment of the surveillance system for HAI.

Methods: Two point-prevalence surveys were performed in 2010 including all inpatients present in the hospital on the days of surveys. HAI was defined as a localized or systemic infection which absent on admission or within the first 48 hours. Logistic regression was applied to study factors associated with HAI.

Results: The prevalence of HAI among all patients (n=604) was 7.3\% (95\% CI: 5.5-9.6). The most common HAIs were upper respiratory tract infections gastroenteritis and pneumonia comprising $38.6 \%, 18.2 \%$ and $15.9 \%$ of cases, respectively. Inverse association between HAI and patients' age was observed, OR=0.82 (95\% CI: 0.72-0.93). Longer stay was associated with higher odds of having HAI. Compared to patients in the neonatal unit, patients in the department of neurology (OR=5.41, 95\% CI: 1.22-23.98) were more likely to have HAI. The predominant HAI in the neurological unit was upper respiratory tract infections while pneumonia and sepsis were most common in the neonatal unit. The use of intravenous (OR=3.56, 95\%CI: $1.1-11.42)$ or urinary (OR=5.42, 95\% CI: $0.71-41.35)$ catheters was positively associated with HAI.

Conclusions: The prevalence of HAI in the hospital more than halved compared to 2006. Prolonged hospital stay, young age, and use of catheters are the factors associated with HAI. Our experience of successful Norwegian-Russian cooperation on establishment of HAI surveillance can be modelled in other Russian hospitals. 


\title{
A5
}

\section{Does the use of snus in adolescence carry an increased risk of entering adult life as a smoker? A study of Norwegian boys followed from age 16 to 19}

\author{
Liv Grøtvedt' ${ }^{1}$ Lisa Forsén², Knut Stavem³, Sidsel Graff-Iversen ${ }^{4}$ \\ ${ }^{1}$ Norwegian Institute of Public Health, Dep. of Health Statistics, Oslo, Norway \\ 2 Norwegian Institute of Public Health, Dep. of Chronic Diseases, Oslo, Norway; Oslo University Hospital, \\ National Competence Centre of Women Health, Oslo, Norway \\ ${ }^{3}$ Akershus University Hospital, Dep. of Pulmonary Medicine, Medical Division, Lørenskog, Norway; Helse \\ Sør-Øst Health Services Research Centre, Lørenskog, Norway; University of Oslo, Faculty of Medicine, \\ Oslo, Norway \\ ${ }^{4}$ Norwegian Institute of Public Health, Dep. of Chronic Diseases, Oslo, Norway; Department of Community \\ Medicine, University of Tromsø, Norway
}

Introduction: The use of moist snuff (snus) in young Norwegians is increasing, while smoking rates are declining. It is not clear whether snus is a gateway to smoking.

Aims: To assess whether boys at 16 years who were never-smokers, but snus users by 2001 baseline, had an increased risk of smoking 3 years later.

Methods: In a prospective school based cohort study, 1,440 boys who responded to a questionnaire in 2001 and 2004 were included for analyses. The participation rate was $89 \%$ in 2001 and $50 \%$ in 2004. Multinomial logistic regression models were used to assess the odds ratio (OR) of snus users, smokers and dual users of cigarettes and snus, compared to non-tobacco-users at baseline, to be smokers at follow-up.

Results: Snus use at baseline was associated with increased odds of dual use at follow-up when the outcome was (1) current dual use vs no tobacco (OR=3.49, 95\% CI 1.8-6.8), and when the outcome was (2) current dual use vs no smoking, but including snus only use $(\mathrm{OR}=1.88,95 \%$ CI 1.1-3.3). In neither model use of snus only at baseline was associated with increased odds of smoking only at follow-up, adjusting for known risk factors.

Conclusions: Snus use may act as a gateway to smoking, as young male snus only users at baseline had an increased risk of being dual users at follow-up. 
A6

\section{Does patterns of maternal use of potentially addictive drugs reproduce in their children?}

Ingeborg Hartz ${ }^{1}$, Thomas Log${ }^{2}$, Randi Selmer ${ }^{3}$, Kari Furu ${ }^{3}$, Aage Tverdal $^{3}$, Eivind Skille 3 , Svetlana Skurtveit ${ }^{3}$

${ }^{1}$ Department of Health and Sports, Hedmark University College, Elverum

${ }^{2}$ University of Troms $\varnothing$

${ }^{3}$ Department of pharmacoepidemiology, Norwegian Institute of Public Health, Oslo

Background: Prevention of use (and abuse) of potentially addictive prescription drugs among young adults presupposes information on factors associated with its use. Epidemiological studies have revealed the importance of social, non-medical and contextual determinants on the use of potentially addictive drugs in general.

Objectives: To investigate the association between maternal use of potentially addictive drugs (benzodiazepines (BZD), z-hypnotics, opioids), maternal socioeconomic characteristics and prescribed use by their offspring.

Methods: The study population was 98,489 young adults who were 15-16 years in 2001, and their mothers, participating in the nationwide Population and Housing Census in Norway in 2001. Data from the census for mother and child were linked to information on use of prescription drugs from the Norwegian Prescription Database (2004-2009). The young adults were 18-24 years during follow-up.

Results: The proportion of the young adults who had retrieved at least one prescription on BZDs/z-hypnotics was $14 \%$ in offspring of maternal users compared to $7.6 \%$ in offspring of maternal non-users, adjusted odds ratio 1.87 (95\% CI 1.79-1.96). There was a positive association between amount of use inn mothers and their offspring. Repeated use of opioids in offspring was positively associated with repeated use in their mothers, adjusted odds ratio 3.1(95\% CI 2.7-3.6). Being a female, lower maternal educational level, mother on disability pension and having a non-married mother were all positively associated with use of BZDs/z-hypnotic use and repeated use of opioids.

Conclusions: Our study suggests that the more extensive use of potentially addictive prescription drugs in mothers, the more extensive use in their children, irrespective of socioeconomic circumstances. However, other maternal markers of a lower socioeconomic position also seemed to independently influence drug use in their offspring. 


\title{
A7
}

\section{Is age of alcohol use initiation the cause of alcohol use disorders in young adulthood? A population-based twin study}

\author{
Eivind Ystrom1, Kenneth S. Kendler $2 ; 3 ; 4$, Ted Reichborn-Kjennerud 1;5;6 \\ ${ }^{1}$ Department of Adult Mental Health, Norwegian Institute of Public Health, Oslo, Norway \\ ${ }^{2}$ Virginia Institute for Psychiatric and Behavioral Genetics, Virginia Commonwealth University School of \\ Medicine, Richmond, USA \\ ${ }^{3}$ Department of Psychiatry, Virginia Commonwealth University School of Medicine, Richmond, USA \\ ${ }^{4}$ Department of Human and Molecular Genetics, Virginia Commonwealth University School of Medicine, \\ Richmond, USA \\ 5Institute of Psychiatry, University of Oslo, Oslo, Norway \\ ${ }^{6}$ Department of Epidemiology, Columbia University, New York, USA
}

Introduction: Age of alcohol use initiation is associated with later alcohol use disorders (AUD), and has been proposed as a cause of later AUD. Both age of alcohol use initiation and AUD have genetic and environmental risk factors. Genetically informative data can indicate to what extent the association is causal or not.

Aims: We wanted to compare two models. First, a model where age of alcohol use initiation is an indicator of genetic or environmental risk for AUD. Thereafter, a mediation model where the genetic and environmental associations are mediated through age of alcohol use initiation. The mediation model is more parsimonious, and should be preferred if it fits no less to the data.

Methods: We utilized a population-based sample of 1336 Norwegian twins who had initiated alcohol use and responded on a clinical interview measuring DSM-IV AUD.

Results: The mediation model had an inferior fit to the data. While 34\% of the observed variation in age of alcohol use initiation was explained by genetic variation, $96 \%$ of the covariation between age of alcohol use initiation was explained by common genetic risk. The remaining $4 \%$ of the covariation was explained by individual-specific environmental risk. Environmental factors shared by siblings explained $(25 \%)$ of the variation in age of alcohol use initiation, but did not explain the covariation with AUD.

Conclusion: Age of alcohol use initiation and AUD appears to have substantially overlapping genetic causes, but appears to a very marginal degree to have overlapping environmental causes. A causal hypothesis on the relationship between age of alcohol use initiation and AUD is not supported by genetically informative data from the Norwegian population. This has clear implications for the potential effect of early interventions against AUD by reducing age of alcohol use initiation. 


\title{
A8 \\ The relationship between unfavourable lifestyle in the ages 13-25 and subsequent use of potentially addictive prescription drugs in Norwegian men and women
}

\author{
Vidar Hjellvik ${ }^{1}$, Marte Handal ${ }^{1}$, Svetlana Skurtveit ${ }^{1}$, Ingeborg Hartz ${ }^{1,2}$ \\ ${ }^{1}$ Norwegian Institute of Public Health, Department of Pharmacoepidemiology, Oslo, Norway \\ ${ }^{2}$ Hedmark University College, Department of Health and Sports, Elverum, Norway
}

Introduction: Early adolescent problem behaviours have in several studies been associated with adult substance use and mental health disorders. However, there is little knowledge about the association between adolescent lifestyle with later inappropriate use of potentially addictive prescription drugs.

Aims: To investigate whether unfavourable lifestyle in adolescents and young adults were related to subsequent use of potentially addictive prescription drugs, and to which extent dropping out of the study was related to lifestyle.

Methods: Self-reported lifestyle data for 2504 adolescents born between 1974 and 1979 who participated in Young in Norway Longitudinal 1992-2005 were linked to the Norwegian Prescription Database 2004-2009. An unfavourable lifestyle indicator $(L)$ ranging from 0 to 4 was calculated based on smoking, alcohol consumption, and illicit drug use in the ages 13-25, and sexual debut age. A drug use index was calculated from yearly consumption of opioids, anxiolytics, and hypnotics. The relative risk of being among the $5 \%$ with the highest drug use index within each gender was calculated for different levels of $L$ with $L=0$ as reference. Adjustments were made for anxiety/depression, source of income, and parents' education, work status, marital status and alcohol consumption. The drop-out rate between 1992 and 2005 was calculated for various levels of the lifestyle variables for 6384 participants in 1992.

Results: Unfavourable lifestyle $(L \geq 3)$ more than doubled the risk of high prescription drug use (relative risk $=2.45$ after adjustment, 95\% confidence interval: 1.50-4.01). Smoking and early sexual debut was the strongest single predictors. Dropping out of the study was strongly associated with all of the lifestyle variables.

Conclusions: Unfavourable lifestyle was positively associated with subsequent use of potentially addictive prescription drugs, and with dropping out of the study. 


\title{
B5
}

\section{Causal inference: Estimation of direct and indirect effects in survival analysis}

\author{
Odd 0. Aalen \\ Department of Biostatistics, Institute of Basic Medical Sciences, University of Oslo
}

Objective: When using survival analysis to study the occurrence of events, one quite often has repeated observations of covariates. For instance, one could study the occurrence of heart disease and have observations of BMI (body mass index) measured at different times throughout the life course of an individual. One might be interested in whether BMI measured at a young age has a separate direct influence on later disease risk, or whether the influence is mediated through the BMI measured at a later age. In other words, could data of this nature be used to say something about mechanisms of disease?

Estimation of direct and indirect effects is an important part of causal inference, and I shall briefly discuss the causal definition of such effects, and the assumptions one must make.

Classical methods of survival analysis have not been well suited to handle this issue. I will present very recent developments with a couple of examples.

This is a methodological talk, presenting results from recent literature. 


\title{
B6
}

\section{Analyzing direct and indirect effects of treatment using dynamic path analysis applied to data from the Swiss HIV Cohort Study}

\author{
Røysland $\mathrm{K}^{1}$, Gran JM11, Ledergerber B2 ${ }^{2}$ Wyl V², Young J3 , Aalen $00^{1}$ \\ ${ }^{1}$ Department of Biostatistics, Institute of Basic Medical Sciences, University of Oslo, Norway \\ ${ }^{2}$ Division of Infectious Diseases and Hospital Epidemiology, University of Zurich, Switzerland \\ ${ }^{3}$ Basel Institute for Clinical Epidemiology and Biostatistics, University Hospital Basel, Switzerland
}

Introduction: When applying survival analysis such as Cox regression on data from major clinical trials or other studies, often only baseline covariates are used. This is typically the case even if updated covariates are available throughout the observation period, which leaves large amounts of information unused. The main reason for this is that such time-dependent covariates often are internal to the disease process, as they are influenced by treatment, and therefore lead to confounded estimates of the treatment effect. There are, however, methods to exploit such covariate information in a useful way.

Aims: The aims of this study is to investigate such methods for estimating direct and indirect effects of treatment, in a setting where treatment is offered at multiple timepoints and so-called time-dependent confounding is present.

Methods: We apply the method of dynamic path analysis to data from the Swiss HIV Cohort Study. To adjust for time-dependent confounding between treatment and the outcome 'AIDS or death', we carry out the analysis on a sequence of mimicked randomized trials constructed from the original cohort data. To analyze these trials together, regular dynamic path analysis is extended to a composite analysis of weighted dynamic path models.

Results: Results using a simple path model, with one indirect effect mediated through current HIV-1 RNA level, show that most, or all, of the total effect go through HIV-1 RNA for the first 4 years. A similar model, but with CD4 level as mediating variable, shows a weaker indirect effect, but the results are in the same direction.

Conclusions: There are reasons to be cautious when drawing conclusions from estimates of direct and indirect effects. Dynamic path analysis is however a useful tool to explore underlying processes, which are ignored in regular analyses. 


\title{
B7
}

\section{The Theory of Causation: Why correlation is not part of causation}

\author{
Rani Lill Anjum \\ Department of Economics and Resource Management, Norwegian University of Life Sciences
}

The philosopher David Hume offered an analysis of causation that has deeply influenced our theoretical causal thinking. According to this analysis, causation is tightly linked to correlation, or what he referred to as constant conjunction. Whenever the cause occurred, the event would typically follow.

Philosophers have been criticising Hume, arguing that correlation is not sufficient for causation: we need something more that ties the cause and its effect together, for instance some necessary connection between them. What has been largely neglected, however, is that correlation is not even necessary for causation. Most, if not all, cases of causation is less than perfect correlation.

The reason for this lies in the very nature of causation: a cause is something that tends towards its effect without guaranteeing it. Smoking tends towards cancer but not everyone who smokes develops cancer. Birth control pills dispose towards thrombosis, but only for about 1 in a 1000 .

This paper aims to spell out some of the basic philosophical features of causation which could explain why causation can never give us correlation or vice versa. It will do so by offering a recently developed theory that challenges the orthodox understanding of causation since Hume. This theory emphasises a variety of features that other theories tend to overlook or pose as problems for causation, such as causal complexity, contextsensitivity and causal interference.

One of the advantages of this theory is that causation becomes a practical rather than a purely theoretical notion. Thus it will also prove more suitable and useful for scientists who deal with the less than idealised situations that are the real-worldly phenomena around us.

S. Mumford and R. L. Anjum: Getting Causes From Powers, Oxford University Press, September 2011. 


\title{
B8
}

\section{Re-use of controls in nested case-control studies with multiple endpoints: A study of prostate cancer and vitamin D}

\author{
Nathalie Støer ${ }^{1}$, Haakon Meyer ${ }^{2,3}$ and Sven Ove Samuelsen ${ }^{1,3}$ \\ ${ }^{1}$ Department of Mathematics , University of Oslo \\ ${ }^{2}$ Department of Community Medicine, University of Oslo, \\ ${ }^{3}$ Norwegian Institute of Public Health
}

Introduction: In a nested case-control study controls are matched to their respective cases. If different endpoints within the same cohort are of interest, the traditional analysis method can only use controls for the current cases in each analysis. Therefore, available information about remaining controls can not be used. However, recently methods have been developed that allow for breaking the matching and thus allow for analysis where the controls are no longer tied to their cases.

We have access to a nested case-control study with the first endpoint being incidence of prostate cancer. However, we also want to analyze the endpoint death of prostate cancer. With the traditional estimator, only controls matched to cases that die of prostate cancer can be included. Since this is only a small subset of all controls (23\%), we loose information from all controls for cases not dying of prostate cancer (77\%). We suggest an alternative method enabling us to use all controls: Calculate the probability of ever being sampled as a control and analyze the data by using inverse probability weighing (IPW).

Aims: The aim is to explore how IPW behave when the controls are matched on more than at risk status. In addition, we explore how much efficiency can be gained by being able to use controls for one endpoint as additional controls for a second endpoint.

Methods: Evaluation of IPW with three different weights was done through analysis on the dataset concerning the association between prostate cancer and vitamin D.

Results: IPW produced estimated relative risks that were similar to the ones estimated with the traditional estimator. In addition, the standard errors were up to $30 \%$ smaller for the death of prostate cancer endpoint.

Conclusions: IPW seems to work quite well when there is additional matching and is a promising method in epidemiology. 
B9

\title{
Selection bias: magnitude and limits
}

\author{
Hein Stigum, Siri Håberg, Anders Skrondal
}

Norwegian Institute of Public Health

Introduction: Selection bias can be represented in a causal graph as collider stratification bias. In its simplest form, bias may arise if selection depends on the exposure and/or the disease. Earlier studies have expressed the size of the bias, but have assumed no interaction between the selection effects.

Aims: To identify selection bias when selection forces interact, and to find limits for the magnitude of bias.

Methods: We examine selection bias in a 2 by 2 table of exposure by disease status. Let $p_{e}$ and $p_{d}$ be the prevalence of exposure and disease respectively, and let RR be the true population effect of exposure on disease. Let $\mathrm{p}$ be the proportion of subjects selected in the absence of exposure and disease (background selection). Let $R R_{e}, R_{d}$ and $R R_{e d}$ be the increase in selection relative to $\mathrm{p}$ for subjects selected due to exposure, disease or the combination of these. We assume a multiplicative selection interaction, and express the selection bias in the relative risk as the RR among the selected divided by the true RR.

Results: The selection bias in the RR is:

$B_{R R}=\frac{\left(1+(R R-1) p_{\theta}+p_{d}\left(R R_{d}-1\right)\right) R R_{\theta d}}{1+(R R-1) p_{\theta}+R R p_{d}\left(R R_{d} R R_{e d}-1\right)}, \quad \lim 1=\frac{R R-1+R R_{d}}{R R R R_{d}}, \lim 2=R R_{e d}$

The bias is bounded by the limits, close to limit 1 for common diseases, close to limit 2 for rare diseases.

The selection bias in the OR is:

$B_{\mathrm{OR}}=\mathrm{RR}_{\mathrm{ed}}$

Conclusion: The derived expressions provide some surprising results: 1) there is no bias created through the mechanism of being selected depending on exposure only (RRe). 2) Bias caused by the selection mechanism involving both exposure and disease ( $\left.R_{\mathrm{ed}}\right)$ has the opposite direction as bias caused by selection dependent on disease only $\left(R_{d}\right)$, and has a stronger effect. These bias mechanisms may cancel out. 3) The bias in the odds ratio is always equal to the selection mechanism involving both exposure and disease $\left(R_{\mathrm{ed}}\right)$. 


\title{
C5
}

\section{European Study of Cohorts for Air Pollution Study (ESCAPE) - updates from Oslo, Norway}

\author{
Christian Madsen ${ }^{1}$, Sveinung Berntsen ${ }^{1}$, Wenche Nystad ${ }^{1}$, Per Erik Schwarze ${ }^{2}$, \\ Per Nafstad 1,3 \\ ${ }^{1}$ Division of Epidemiology, Norwegian Institute of Public Health, Oslo, Norway \\ 2 Division of Environmental Medicine, Norwegian Institute of Public Health, Oslo, Norway \\ ${ }^{3}$ Department of General Practice and Community Medicine, University of Oslo, Norway
}

Introduction: The European Study of Cohorts for Air Pollution Effects (ESCAPE) is a large EU-funded study on long-term health effects of outdoor air pollution. The study uses land use regression (LUR) modeling to add air pollution exposure to health data already available from European cohort studies. The results will be used to revise the different air pollution standards in the European Union.

Aims: Develop local models that combine both the spatial and the temporal distribution traffic-related pollution ( $\mathrm{PM}_{10}, \mathrm{PM}_{2.5}$, the soot content of $\mathrm{PM}_{2.5}$ and $\mathrm{NO}_{\mathrm{x}}$ ) and use these to predict historical exposure using geocoded addresses for participants in the area.

Methods: 40 sites were selected to represent the distribution of air pollution in the Oslo area. These were sampled for two-weeks once during winter, spring and summer using Ogawa badges and Harvard impactors. In addition we chose one continuous sampling site to measure all year for adjustment of temporal variations in the final data. The measurements was then included as averaged annual values to develop stochastic models using predictor variables (e.g. traffic, population, topography, land use) using Geographic Information Systems (GIS). Available monitoring data was included in the final models to back-extrapolate the spatial distribution. Additional samples have been analyzed by x-ray fluorescence (XRF) to determine their elemental composition by source group.

Results: The observed concentrations showed good variation by site type (regional background, urban background, and urban traffic). The models included several traffic-related predictors and explained between 66-83 percent of the observed annual variance in air pollution.

Conclusions: LUR models were developed which explained a large fraction of variability, with road traffic being the most important predictor. 
C6

\title{
Tools to facilitate inter-disciplinarity in environmental health decision making
}

\author{
Alena Bartonova, Hai-Ying Liu, Sonja Grossberndt and Aileen Yang \\ NILU Norwegian Institute for Air Research, Kjeller
}

\begin{abstract}
Introduction: Requirements on scientists to work in highly inter-disciplinary environment, and on their ability to communicate with decision makers, grow. The FP6 coordination action Health and Environmental Network (HENVINET) aimed to facilitate collaboration, by creating a permanent network of professionals in environment and health, with tools and methods supporting an interdisciplinary environment. Traditional scientific instruments do not satisfy the needs for informing the society. A broad consensus is required, together with traceable knowledge pedigree, for knowledge to be used. A common framework is essential. An active involvement of broad range of experts, and identification and assessment of agreements and disagreements, and collaboration tools to enable decision making are needed.
\end{abstract}

\begin{abstract}
Aims: The aim of this work was to facilitate inter-disciplinary collaboration in environment and health, and to create a professional network.

Methods: We chose the Drivers-Pressures-State-Exposure-Effect-Action framework as a structuring method for information gathering and collaboration. Interactive web based tools and methods for knowledge evaluation were taken forward towards formulating implications for policy. Open architecture web technology enabled searchable databases and web portal.
\end{abstract}

Results: Dedicated web based tool for knowledge evaluation allowed remote expert elicitation, used as a basis for development of policy advice in five areas (asthma and allergies, cancer, neurodevelopment disorders and endocrine disruption, engineered nano-particles in the environment). An open searchable database of decision support tools was established. A web based social networking tool is now available for networking.

Conclusions: Inter-disciplinary collaboration and communication are the main challenges in informed decision making and in creating a network in environment and health. The project has developed a variety of methods and tools, and has produced scientifically based politically relevant advice. It has increased the awareness of the need to go beyond traditional scientific tools and methods. The tools and experiences can be carried forward to improve environmental health governance. 


\title{
C7
}

\section{The short-term effect of 24-hour average and peak air pollution on mortality in Oslo, Norway: A case-crossover analysis of neighbourhood exposure and individual effect modifiers}

\author{
Christian Madsen ${ }^{1}$, Pål Rosland ${ }^{2}$, Dominic A. Hoff ${ }^{1}$, Wenche Nystad ${ }^{1}$, Per Nafstad ${ }^{1,3}$ \\ Øyvind E. Næss ${ }^{1}$ \\ ${ }^{1}$ Division of Epidemiology, Norwegian Institute of Public Health, Oslo, Norway \\ ${ }^{2}$ Norwegian Public Road Administration, Oslo, Norway \\ ${ }^{3}$ Department of General Practice and Community Medicine, University of Oslo, Norway
}

Introduction: Numerous epidemiological studies have shown associations between increases in outdoor air pollution and all-cause mortality as well as cardiovascular and respiratory related mortality. Most studies have used monitoring stations and thus have not been able to separate daily averages and local variations in peak-values within urban settings.

Aims: Explore short-term associations between outdoor air pollution on mortality comparing 24-hour averages to peak concentrations (during rush hours) of nitrogen dioxide $\left(\mathrm{NO}_{2}\right)$ and particulate matter $\left(\mathrm{PM}_{2.5}\right)$ at residential neighbourhood level.

Methods: We used a time-stratified case-crossover design to estimate association of traffic-related air pollution and wood-burning with daily mortality during a period of 10 years (1992-2001) among residents above 50 years of age in Oslo, Norway. A dispersion model was used to assess short-term air pollution for 24-hour averages and peak concentrations of nitrogen dioxide $\left(\mathrm{NO}_{2}\right)$ from exhaust and particulate matter $\left(\mathrm{PM}_{2.5}\right)$ from exhaust and wood-burning at residential neighbourhood level for each individual.

Results: The short-term for 24-hour average concentrations at lag 0-5 indicated an increased risk per $10 \mu \mathrm{g} / \mathrm{m}^{3}$ change in exposure of 0.6 percent (95\% CI: -1.1 to 2.3 ) for $\mathrm{NO}_{2}$ and 2.8 percent (95\% CI: 1.2 to 4.4 ) for $\mathrm{PM}_{2.5}$. The short-term for average peak concentrations at the same lag indicated an increased risk per $10 \mu \mathrm{g} / \mathrm{m}^{3}$ change in exposure of 1.5 percent ( $95 \%$ CI: 0.4 to 2.6) for $\mathrm{NO}_{2}$ and 1.5 percent (95\% CI: 0.6 to 2.4) for $\mathrm{PM}_{2.5}$.

Conclusions: Short-term traffic-related air pollution is associated with increased risk for mortality among individuals above 50 years of age, especially for circulatory outcomes. The 24-hour average is describing a higher risk from $\mathrm{PM}_{2.5}$ exposure due to other sources, while the peak concentrations gives an overall increased risk for both $\mathrm{NO}_{2}$ and $\mathrm{PM}_{2.5}$ for the most trafficated periods of the day. 
C8

\section{Is the quality of drinking water a risk factor for forearm fractures? Cohort of Norway}

Cecilie Dahl ${ }^{1,2 *}$, Anne Johanne Søgaard ${ }^{1}$, Grethe S Tell ${ }^{2}$, Trond Peder Flaten ${ }^{3}$, Truls Krogh4, Geir Aamodt ${ }^{1}$. On behalf of the NOREPOS Research Group

${ }^{1}$ Division of Epidemiology, Norwegian Institute of Public Health, Oslo, Norway

${ }^{2}$ Department of Public Health and Primary Health Care, University of Bergen, Bergen, Norway

${ }^{3}$ Department of Chemistry, Norwegian University of Science and Technology, Trondheim, Norway

${ }^{4}$ Division of Environmental Medicine, Norwegian Institute of Public Health, Oslo, Norway

Introduction: The Norwegian population experiences fractures at a rate that is among the highest ever reported. A large variation in fracture rate between and within countries indicates that an environmental factor, such as the quality of drinking water, could be one of the causes of the disparities. Our aim was to investigate a possible association between $\mathrm{pH}$ (an important parameter for water quality) and self-reported forearm fracture, and to examine whether other water quality factors could account for this association.

Methods: Using Geographic Information Systems, information on the quality of drinking water was linked to participants in Cohort of Norway $(n=173,243)$, a database comprising ten regional epidemiological health surveys from across the country in the time period 1994-2003.

Results: The highest risk of forearm fracture was at $\mathrm{pH} 6.75$, with a decreasing risk toward both higher and lower $\mathrm{pH}$ values. Categorizing $\mathrm{pH}$ into two groups $(<7.5$ and $\geq 7.5)$ based on national recommendations for $\mathrm{pH}$ in drinking water, we saw an increased odds of forearm fracture in men consuming water with $\mathrm{pH}<7.5$ compared to $\mathrm{pH} \geq 7.5(\mathrm{OR}=1.20$, 95\% CI: 1.14, 1.27), and a corresponding increased odds in women (OR=1.14, 95\% CI: $1.09,1.20)$. This association was attenuated after adjustment for other water quality factors (color grade, intestinal enterococci and Clostridium perfingens).

Conclusions: Our findings indicate a higher risk of fracture when consuming water with a pH less than 7.5, however the risk does not seem to be due to the acidity level per se, but rather to other aspects of water quality associated with $\mathrm{pH}$. 


\title{
A9
}

\section{Comparison of land-use regression models for predicting spatial $\mathrm{NO}_{\mathrm{x}}$ contrasts over a three year period in Oslo, Norway}

\author{
Christian Madsen ${ }^{1}$, Ulrike Gehring2 ${ }^{2}$, Siri Eldevik Håberg1', Per Nafstad ${ }^{1,3}$, Kees Meliefste², \\ Wenche Nystad ${ }^{1}$, Karin C. Lødrup Carlsen ${ }^{4}$, Bert Brunekreef 2,5 \\ ${ }^{1}$ Division of Epidemiology, Norwegian Institute of Public Health, Oslo, Norway \\ ${ }^{2}$ The Institute for Risk Assessment Sciences (IRAS), Utrecht University, The Netherlands \\ ${ }^{3}$ Department of General Practice and Community Medicine, University of Oslo, Norway \\ ${ }^{4}$ Department of Paediatrics, Woman and Child Division, Oslo University Hospital and the Faculty of \\ Medicine, Oslo, Norway \\ ${ }^{5}$ Julius Center for Health Sciences and Primary Care, University Medical Center Utrecht, The Netherlands
}

Introduction: Spatial modelling of traffic-related air pollution through land-use regression (LUR) is increasingly applied in epidemiological studies. These models provide highly spatially resolved data, but assume that the spatial contrasts are stable over long periods of time. It is not known to which extent these models can be used to predict concentrations in earlier or later periods.

Aims: Testing the stability of measured and modelled spatial contrasts over a three year period in order to assess the relevance for future assessments of individual exposure to traffic-related air pollutants in epidemiological studies.

Methods: A land-use regression model was previously developed to estimate addresslevel outdoor concentrations of traffic-related air pollution based on samples of nitrogen oxides $\left(\mathrm{NO}_{\mathrm{x}}\right)$ at 80 locations during the winter of 2005 . These locations were re-sampled using the same protocol three years later (69 of 80 locations) and used to develop a new LUR model.

Results: Measurements conducted in 2008 agreed well with measurements sampled in 2005 at the same locations $(r=0.91-0.95)$. The LUR models from 2005 and 2008 explained $66-77 \%$ and $60-74 \%$ of the variability of the measured concentrations, respectively. The 2008 LUR models explained $55-68 \%$ of the spatial variability of the 2005measurements, while the 2005 LUR models explained $53-66 \%$ of the spatial variability of the 2008-measurements. The models performed better for $\mathrm{NO}_{\mathrm{x}}$ and $\mathrm{NO}_{2}$ compared to $\mathrm{NO}$, and were shown to be equally valid when using leave-one-out cross-validation and validation of models based on independent training sets.

Conclusions: We found a good agreement between short-term measured spatial contrasts in outdoor $\mathrm{NO}_{\mathrm{x}}$ over a three year period. LUR models for this area performed equally well using two different validation methods. These models predicted the spatial variation well for this area both forward and backward in time. 


\title{
A10
}

\section{Road traffic noise, air pollution and hypertension in a population-based sample in Oslo}

\author{
Bente Oftedal ${ }^{1}$, Sviatlana Panasevich ${ }^{1}$, Per Nafstad ${ }^{2,3}$, Per E. Schwarze ${ }^{1}$, \\ Gunn Marit Aasvang ${ }^{1}$ \\ ${ }^{1}$ Norwegian Institute of Public Health, Division of Environmental Medicine, Oslo, Norway \\ ${ }^{2}$ University of Oslo, Institute of Health and Society, Oslo, Norway \\ ${ }^{3}$ Norwegian Institute of Public Health, Division of Epidemiology, Oslo, Norway
}

Introduction: Epidemiologic studies have reported that exposure to traffic-related noise as well as air pollution is associated with hypertension and cardiovascular pathology. However, the knowledge about the relative importance of noise and air pollution in developing cardiovascular diseases is still limited.

Aims: To examine the relationship between residential road traffic noise, air pollution and hypertension.

Methods: Adult participants of the population-based Oslo Health Study (HUBRO) $(\mathrm{N}=8,921)$ from 2000-2001 were assigned exposure estimates for residential road traffic noise at most exposed façade and nitrogen dioxide $\left(\mathrm{NO}_{2}\right)$ levels. The noise indicators $\mathrm{L}_{\mathrm{den}}$ and $L_{\text {night }}$ for the year 2006 were calculated on $5 \times 5 \mathrm{~m}^{2}$ grid, using The Nordic Prediction Method. Future analyses will include noise exposure estimates for the year 2000, providing data at the time of conducting HUBRO. $\mathrm{NO}_{2}$ exposure was estimated by the EPISODE dispersion model mainly on $1 \mathrm{~km}^{2}$ grid. Hypertension was defined as measured systolic blood pressure above $140 \mathrm{mmHg}$, measured diastolic blood pressure above $90 \mathrm{mmHg}$ or self-reported use of antihypertensive medication.

Results: The correlation between road traffic noise and $\mathrm{NO}_{2}$ was 0.4 . Preliminary results showed an odds ratio (OR) of 1.03 for hypertension (95\% confidence interval [CI] 0.971.09) per interquartile range (IQR) higher level of traffic noise ( $\mathrm{L}_{\text {night}}$ ) adjusted for age, gender, body mass index and education. No association was found between $\mathrm{NO}_{2}$ and hypertension. Adjusting for $\mathrm{NO}_{2}$ did not change the OR of traffic noise. In men we found an OR of 1.06 (95\% CI 0.97-1.15) per IQR increase of traffic noise (Lnight), and OR=0.99 (95\% CI 0.91-1.08) in women.

Conclusions: Residential road traffic noise may not be related to hypertension among participants of HUBRO. The association was somewhat stronger in men than women, but did not reach statistical significance. These associations are probably not affected by traffic-related air pollution in Oslo. 


\title{
A11
}

\section{Water supply and prevalence of ulcerative colitis using Cohort of Norway and the Norwegian waterworks registry}

\author{
Geir Aamodt 1,2, May-Bente Bengtson ${ }^{3}$, Cecilie Dahl ${ }^{1}$, Truls Krogh ${ }^{4}$, Morten H. Vatn ${ }^{5,6}$ \\ ${ }^{1}$ Norwegian Institute of Public Health, Division of Epidemiology, Oslo, Norway \\ 2 Norwegian University of Life Sciences, Department of Mathematical Science and Technology, Ås, Norway \\ 3 Tønsberg Hospital, Medical Department, Tønsberg, Norway \\ ${ }^{4}$ Norwegian Institute of Public Health, Division of Environmental Medicine, Oslo, Norway \\ 5 The Institute of Clinical Epidemiology and Molecular Biology (EpiGen), Faculty Division Akershus \\ University Hospital, Nordbyhagen, University of Oslo, Norway \\ ${ }^{6}$ Medical Clinic, Oslo University Hospital, Rikshospitalet, Oslo, Norway
}

Introduction: Ulcerative colitis is characterized by a dysfunction of the intestinal epithelium barrier, resulting in chronic inflammation. It is assumed that the disease is caused by an imbalance between the immune system and the commensal microbial flora in genetically susceptible individuals. The quality of drinking water has been reported as a risk factor for ulcerative colitis in Norway. Iron was the strongest component, but $\mathrm{pH}$, color, turbidity, and coliform bacteria were also associated with the development of the disease.

Aims: We wish to study associations between components in drinking water and the prevalence of ulcerative colitis. In addition, we will investigate if typical patterns in the drinking water, identified using principal component analysis, are associated with the disease.

Methods: We used prevalent cases of ulcerative colitis identified from self-reported medication use in the Cohort of Norway database; a collection of health surveys conducted from 1994 to 2003 in 7 out of 19 counties. The total number of participants was 81,189, and we identified 360 individuals with ulcerative colitis. We utilized water quality information from the Norwegian waterworks registry and linked it to the participants home addresses using geographic information systems. Different imputations techniques were used to handle missing observations.

Results: Many components in purified drinking water are associated with ulcerative colitis such as $\mathrm{pH}$, color grade, aluminum, and microbiological components. The results from principal component analysis showed that the first two factors spanning the variability among the water components, were associated with ulcerative colitis.

Conclusions: Water supply and drinking water quality are associated with the development of ulcerative colitis. Many single components are associated with the disease, but the composition or pattern of the drinking water could be of more importance than the single components. Drinking water quality could show to be an important factor in determining the gut flora. 


\title{
A12
}

\section{Hip fracture incidence in Norway 1999-2008. Norwegian Epidemiologic Osteoporosis Studies}

\author{
Tone K. Omsland ${ }^{1,2}$, Kristin Holvik1,2, Nina Emaus ${ }^{2,3}$, Grethe Tell2, Berit Schei ${ }^{4,5}$, \\ Aage Tverdal ${ }^{1}$, Clara Gram Gjesdal ${ }^{6}$, Guri Grimnes ${ }^{7}$, Siri Forsmo ${ }^{5}$, Haakon Meyer ${ }^{1,8}$, \\ Anne Johanne Søgaard ${ }^{1}$ \\ ${ }^{1}$ Division of Epidemiology, Norwegian Institute of Public Health, Oslo, Norway \\ ${ }^{2}$ Department of Public Health and Primary Health Care, University of Bergen, Norway \\ ${ }^{3}$ Department of Health and Care Sciences, University of Tromsø, Troms $\emptyset$, Norway \\ ${ }^{4}$ Institute of Public Health and General Practice/Department of Obstetrics and Gynecology, Faculty of \\ Medicine, St. Olav's University hospital, Trondheim, Norway \\ ${ }^{5}$ Institute of Public Health and General Practice, Faculty of Medicine, Norwegian University of Science and \\ Technology (NTNU), Trondheim, Norway \\ ${ }^{6}$ Department of Rheumatology, Haukeland University Hospital, Bergen, Norway \\ ${ }^{7}$ Division of Internal Medicine, University Hospital of North Norway, Tromsø, Norway \\ ${ }^{8}$ Institute of Health and Society, Department of Community Medicine, University of Oslo, Norway
}

Background: Hip fractures constitute a major health problem in the elderly. A decline in hip fracture rates has recently been reported in many Western countries. Norway has among the highest hip fracture rates in the world.

Aim: To study time trends in hip fracture incidence in Norway from 1999 to 2008.

Methods: All cervical, trochanteric and sub-trochanteric hip fractures treated in Norwegian hospitals with a diagnosis code for hip fracture (ICD 9: 820 with all subgroups; ICD 10: S72.0-S72.2) between 1994 and 2008 were retrieved through a system developed by the Norwegian Knowledge Centre for the Health Services. Additional diagnosis codes and surgical procedure codes were available for all hospitalisations. Based on these codes and time between hospital stays, hospital admissions for primary hip fractures were identified.

The individual's first hip fracture was counted. To avoid misclassification of second hip fractures as first hip fracture, we started counting the first hip fractures in 1999, as the majority of refractures occur within five years. The estimated misclassification of second fractures after 1999 was less than 2\%. The database named the NOREPOS (Norwegian Epidemiologic Osteoporosis Studies) hip fracture database was validated against local hip fracture registries in Troms $\emptyset$ and Oslo. The accuracy of the NOREPOS database varied from $-2 \%$ to $+1 \%$ (Cohen`s kappa=0.95).

Results: Among men and women 50 years and older, a total of 81,891 subjects sustained their first hip fracture between 01.01.1999 and 31.12.2008 - i.e. approximately 8,200 hip fractures per year. The age standardized hip fracture rate declined by $7 \%(p=0.001)$ in men and 19\% $(\mathrm{p}<0.001)$ in women during the 10-year period. The decline was largest in the oldest age groups in women.

Conclusion: A significant decline in hip fracture rates was observed during the 10-year period. The decline was largest in women. 


\title{
B10
}

\section{Weight cycling increase the risk of forearm fractures in men. The Tromsø Study}

\author{
Anne Johanne Søgaard 1, Nina Emaus 2,3 \\ ${ }^{1}$ Division of Epidemiology, Norwegian Institute of Public Health, Oslo, Norway \\ ${ }^{2}$ Department of Health and Care Sciences, University of Tromsø, Tromsø, Norway \\ ${ }^{3}$ Department of Public Health and Primary Health Care, University of Bergen, Bergen, Norway
}

Introduction: A large proportion of the Western population is attempting to lose weight at any age. The consequences of repeated loss and regain of weight on bone is unknown. Findings from the Oslo Study (1972/73-2000) indicate that dieting, both number of times and amount of weight loss, is associated with increased risk of self-reported forearm fractures in younger men. Data from women is lacking.

Aims: This study examines if weight cycling is a risk factor for forearm fractures in women and men.

Methods: In 1994/95 weight and height were measured in all participants aged 25-69 years in the Troms $\varnothing$ Study (14,166 women, 12,791 men). Information was collected about socio-economic background, diseases, lifestyle factors, self-reported forearm fractures, number of dieting episodes and amount of weight lost from the age of 20 or later. Weight loss was divided into four categories: $0,1-5,6-10$ and $11+\mathrm{kg}$ and number of dieting episodes were grouped into: $0,1-5,6-10$ and $11+$ episodes. Logistic regression models were used to estimate odds ratio (OR) of fractures with $95 \%$ confidence interval (CI).

Results: 1087 (10.5\%) women and 1297 (13.5\%) men reported to have suffered a forearm fracture. In men there was an increasing risk of forearm fracture with increasing number of $\mathrm{kg}$ weight loss ( $\mathrm{p}$ for trend $=0.003$ ) and increasing number of dieting episodes ( $\mathrm{p}$ for trend $=0.024$ ) adjusted for covariates. Men reporting weight loss of more than 10 $\mathrm{kg}$ had $\mathrm{OR}=1.49$ (95\% CI 1.15-1.93) for forearm fracture compared to $0 \mathrm{~kg}$. Compared to 0 dieting episodes, those who reported more had an OR=1.19 (95\% CI 1.03-1.39) for forearm fracture. No corresponding associations were observed in women.

Conclusion: Weight cycling - both amount of weight loss and number of dieting episodes, is associated with increased risk of forearm fractures, but only in men. 


\section{B11}

\section{Does instrument error impact the estimated prevalence of overweight and obesity in population-based surveys?}

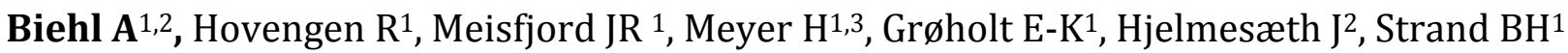

1. Division of Epidemiology, Norwegian Institute of Public Health, Oslo, Norway

2. The Morbid Obesity Centre, Vestfold Hospital Trust, Tønsberg, Norway

3. Institute of Health and Society, Faculty of Medicine, University of Oslo, Oslo, Norway

Introduction: It is important to ensure high quality of surveillance data to follow trends and to compare the estimated prevalence of overweight and obesity. This study is based on the fact that instrument error increases the variance of the BMI distribution, and in combination with cut-off value this will likely impact the estimated proportion of overweight and obesity. Attention to implications that uncalibrated instruments might have is limited.

Aim: To assess the impact of instrument error, due to uncalibrated scales and stadiometers, on the estimated prevalence of overweight and obesity.

Methods: Anthropometric measurements from a nationally representative sample, The Norwegian Child Growth Survey (CGN) of 3474 children, were used. Each of the 127 participating schools received a reference weight and length to determine the correction value. Correction value corresponds to instrument error and is the difference between the true value and the measured, uncorrected weight and height at the local scales and stadiometers. Simulations were used to determine the expected implications of instrument errors. To systematically investigate this, the coefficient of variation (CV) of instrument error was used in the simulations and was increased successively.

Results: Simulations showed that the estimated prevalence of overweight and obesity increased systematically with the size of instrument error. The estimated prevalence of overweight and obesity was $16.4 \%$ with no instrument error. Simulations showed that the estimated prevalence was overestimated by 0.5 percentage point based on observed variance of instrument error from the CGN-study and mean set to zero. Further, the estimated prevalence was $16.7 \%$ with $1 \% \mathrm{CV}$ of instrument error, and increased to $17.8 \%$ with $2 \% \mathrm{CV}, 19.5 \%$ with $3 \% \mathrm{CV}$ and to $21.6 \%$ with $4 \% \mathrm{CV}$.

Conclusions: To our knowledge this is the first study that shows that failure to calibrate may lead to overestimation of the prevalence of overweight and obesity in populationbased surveys. 


\title{
B12
}

\section{Agreement between self-reported data on medicine use in the Norwegian Mother and Child cohort study and prescription records}

\author{
S. Skurtveit, R. Selmer, Aa. Tverdal, K. Furu, W. Nystad and M. Handal \\ Norwegian Institute of Public Health, Division of Epidemiology, P.O.Box 4404 Nydalen, Oslo, Norway
}

Introduction: Most women use prescribed drugs during pregnancy. Use of drugs during pregnancy is complicated because of the limited knowledge with regard to beneficial and possible adverse effects for both the mother and the fetus.

Aims: To study self- reported drug use (opioids, antidepressants and benzodiazepines), among pregnant women in The Norwegian Mother and Child cohort study (MoBa) and to compare this information with drug use recorded in Norwegian Prescription Database (NorPD).

Methods: The study is based on MoBa and NorPD. Data from MoBa on the use of opioids, antidepressants and benzodiazepines reported by pregnant women were compared with data on dispensed medicaments recorded in NorPD. The study population consisted of 28479 women who participated in MoBa and answered all questionnaires in pregnancy and pregnancy started after March 1 2004. Data on dispensed drug were extracted for three different fixed time windows: a) the pregnancy period b) 30 days prior to pregnancy in addition to pregnancy c) 60 days prior to pregnancy in addition to pregnancy. Agreement was assessed by the kappa statistic. Data on dispensed drugs as recorded in NorPD were used as the reference standard in validity analysis. Sensitivity, specificity, positive and negative predictive values were calculated.

Results: Highest kappa, 0.69, was observed for antidepressants, rising to 0.72 when the time window for dispensed drugs was extended by 30 days prior to pregnancy. Agreement was fair for benzodiazepines anxiolytics and hypnotics and opioids (kappa around 0.40). Sensitivity was highest for antidepressants and benzodiazepine-antiepileptics: $72 \%$ of patients with a prescription record of antidepressants and $89 \%$ of patients with a prescription record of benzodiazepine-antiepileptic during pregnancy, reported use on the questionnaires. Specificity was above $97 \%$ for all drugs.

Conclusion: Self-reported use of antidepressants and benzodiazepine-antiepileptic drugs had high sensitivity and specificity. Lower sensitivity and agreement was observed for opioids, benzodiazepine-hypnotics and anxiolytics users. 


\title{
B13
}

\section{Smoking prevalence in the "Smoking, Women and Cancer Study" compared to smoking prevalence in the official Norwegian statistics}

\author{
Eivind Bjerkaas (1), Ranjan Parajuli (1), Elisabete Weiderpass Vainio (1,2,3), \\ Inger Torhild Gram (1). \\ (1) Department of Community Medicine, University of Tromsø, Tromsø, Norway \\ (2) The Cancer Registry of Norway, Oslo, Norway \\ (3) Karolinska Institutet, Department of Medical Epidemiology and Biostatistics, Stockholm, Sweden
}

Introduction: One objective in the "Smoking, Women and Cancer Study" is to study smoking prevalence and breast cancer incidence. The study period is between 1974 and 2003 and includes approximately 330000 Norwegian women from 35 different Norwegian health surveys. We would like to access if the smoking prevalence in the study database reflects smoking prevalence in the official Norwegian statistics for women of the same age group and time period.

Aims: To examine if smoking prevalence in the "Smoking, Women and Cancer Study" is comparable with smoking prevalence obtained from estimates provided by the Statistics Norway for the same age groups and periods. If smoking information is comparable in these data sources, studies on smoking and cancer risk based on the study data can be extrapolated to the entire Norwegian population (external validity).

Methods: Our study includes "Three Counties" (1974, n=45 980), "40 Years Study" (1985, $n=210$ 889), "CONOR" (1994, $n=73$ 472). These different studies were conducted with different aims and intentions, and the questionnaires were not similar until the foundation of "CONOR" in 1994. We have merged different variables into one large database, and linked our data to the Norwegian Cancer Registry and Death Registry. Our main smoking variable is smoking status (never, former, current).

Results: The prevalence of current smokers among women in our database is 39\%. According to the official statistics, the smoking prevalence is $31 \%$ for the same time period (age 16-74). There are large variations among different age groups and time periods.

Conclusions: Smoking prevalence in the study database is in accordance with smoking prevalence in the official Norwegian statistics. Analysis based on the study database can therefore originate results that can be representative for the general Norwegian population. 


\title{
C9
}

\section{Register prevalence of autism spectrum disorders, ADHD, epilepsy and cerebral palsy in Norwegian children}

\author{
Pål Surén 1, 2, Inger Johanne Bakken 3 ${ }^{3}$, Heidi Aase 2, Richard Chin 1, 2, 4 , Nina Gunnes ${ }^{2}$, \\ Kari Kveim Lie ${ }^{2}$, Ted Reichborn-Kjennerud ${ }^{2}$, Synnve Schjølberg ${ }^{2}$, Anne-Siri Øyen ${ }^{2}$, \\ Camilla Stoltenberg 2 \\ 1) Centre for Paediatric Epidemiology and Biostatistics, UCL Institute of Child Health, London, United \\ Kingdom \\ 2) The Norwegian Institute of Public Health, Oslo, Norway \\ 3) The Norwegian Patient Registry, Trondheim, Norway \\ 4) Muir Maxwell Childhood Epilepsy Research Centre, Child Life and Health, University of Edinburgh, \\ Edinburgh, United Kingdom
}

Introduction: Neurological and neurodevelopmental disorders account for a considerable proportion of long-term disabilities in children. Hence, monitoring the population prevalence of the disorders is important. Few studies have investigated the combined prevalence of these disorders, and the extent of overlap between the diagnoses is largely unknown.

Aims: The aim of the study was to determine the proportion of children aged $0-11$ years with one or more of the diagnoses autism spectrum disorder (ASD), attention-deficit hyperactivity disorder (ADHD), cerebral palsy (CP) and epilepsy; and the ratio between boys and girls with these diagnoses.

Methods: Data on ASD, ADHD, CP and epilepsy in children born 1999-2010 was obtained from the nationwide Norwegian Patient Registry (NPR) for the years 2008-2010. The registry contains diagnoses assigned by Norwegian specialist healthcare services (hospitals and outpatient clinics) and (from 2008 onwards) also the Norwegian 11-digit personal identification number of the patients. Information on the total population of children in Norway born 1999-2010 was obtained through Statistics Norway.

Results: For ASD, ADHD and epilepsy the registry prevalence increased with age. At age eleven it was $0.70 \%$ for ASD, $2.87 \%$ for ADHD, and $0.94 \%$ for epilepsy. Prevalence of CP was stable around $0.3 \%$ between age six and eleven. For all the disorders boys had a higher prevalence than girls. Male:female ratios were 4.3 for ASD, 2.9 for ADHD, 1.2 for epilepsy, and 1.3 for CP. There was considerable overlap between the diagnoses - most pronounced between CP and epilepsy.

Conclusion: ASD, ADHD, CP and epilepsy together represent a significant burden of disease in children, and that burden is disproportionately skewed towards boys. The overlap between the disorders may indicate similarities in causal pathways. 


\title{
C10
}

\section{The Norwegian Child Growth Study: Trends in height, weight and waist circumference among third graders (8-9 yrs), 2008-2010}

\author{
Ragnhild Hovengen, Anna Biehl, Jørgen Meisfjord, Bjørn Heine Strand \\ Department of Health Statistics, Norwegian Institute of Public Health, Oslo, Norway
}

Introduction: Childhood obesity and overweight are rapidly increasing world wide, and represent a growing threat to the health in childhood and later life. In Norway a study from Bergen shows that overweight and obesity among children have increased over the last three decades. However, The Norwegian Child Growth Study started in 2008 and is the only nationwide study monitoring growth trends among school children.

\begin{abstract}
Aims: The main aim is to document the status of height, weight, body mass index (BMI) and waist circumference among third graders, as well as to monitor the national and regional growth trends in this age group.
\end{abstract}

Methods: A stratified two-stage sampling design was applied, where the primary sampling unit was counties $(n=10)$ and the secondary sampling unit was the school (127 of 3920 public primary schools). 3500 third graders participated; the response rate was $90 \%$. The variable identifying the geographical strata was the administrative Health Region.

Results: The estimated proportion of overweight and obesity combined increased from 16 to 19 per cent in the period 2008-2010. The increase was seen among both girls and boys. Girls had a higher proportion with overweight and obesity than boys in both years, 17 versus 14 per cent in 2008 ( $\mathrm{p}=0.02)$ and 22 versus 17 per cent in 2010 ( $\mathrm{p}=0.03)$. Health Regions North and Central had the highest proportion of overweight and obesity in both years.

Conclusions: Girls had a higher proportion of overweight and obesity than boys, but the increase over time was of the same magnitude in both girls and boys. However, we only have results from two time points, so it might be too early to conclude whether this increase is an expression of a long time trend in overweight and obesity among Norwegian children. In autumn 2012 the survey will be conducted again by the same schools. 


\title{
C11
}

\section{Effects of preventive mental health programs in schools: A longitudinal quasi experimental intervention study with Solomon's design}

\author{
Bror Just Andersen, Erik Nord, Rune Johansen
}

The research propose of this PhD-project is to evaluate the effects of a preventive mental health program for youth (VIP), in short and long term.

VIP is a preventive mental health program in Norway for students in secondary school. It aims at increasing understanding and recognition of mental problems and lowering thresholds for help seeking.

I've quantified the interventions effects on the following outcome variables:

- The pupil's knowledge about metal health

- Their ability to recognize signals of mental health problems

- The youths help seeking behavior

- Mental health (self-reported with SDQ-Nor)

I've assessed the degree of achievement of the interventions goals. A sample of 880 students in a county where the program had been implemented was compared with a sample of 811 students in a county where the program had not yet been implemented. Data was collected through questionnaires prior to intervention (t0) and at $1(\mathrm{t} 1), 6,12$ and 24 months after intervention.

At each time, knowledge was measured as percentages of top scores on a set of indices. Effect sizes on the various indices are estimated in terms of (a) differences in improvements of percentage scores and (b) Cohen's d. From t0 to t1, t2 and t 3 the intervention group showed significantly greater progress in most of the knowledge indexes and after 12 months we also found significant effects on several mental health indexes.

Compared with effect sizes from similar studies the VIP-program seems to lie in the higher end of the scale. 


\title{
C12
}

\section{Polymorphisms in the innate immune IFIH1 gene, frequency of entero- virus in monthly fecal samples during infancy, and islet autoimmunity}

\author{
Kjersti S. Rønningen ${ }^{4}$ \\ ${ }^{1}$ Norwegian Institute of Public Health, Oslo, Norway \\ ${ }^{2}$ Second Faculty of Medicine, Charles University Prague, The Czech Republic \\ ${ }^{3}$ Glostrup Research Institute, Glostrup, Denmark \\ ${ }^{4}$ Oslo University Hospital, Rikshospitalet, Oslo, Norway
}

Elisabet Wits $\varpi^{1}$, German Tapia ${ }^{1}$, Ondrej Cinek $^{2}$, Flemming Pociot ${ }^{3}$, Lars C. Stene ${ }^{1}$ and

Introduction: Interferon induced with helicase C domain 1 (IFIH1) senses and initiates antiviral activity against enteroviruses. Genetic variants of IFIH1, one common and four rare SNPs have been associated with lower risk for type 1 diabetes.

Aims: Our aim was to test whether these type 1 diabetes-associated IFIH1 polymorphisms are associated with the occurrence of enterovirus infection in the gut of healthy children, or influence the lack of association between gut enterovirus infection and islet autoimmunity.

Methods: After testing of 46,939 Norwegian newborns, 421 children carrying the high risk genotype for type 1 diabetes (HLA-DR4-DQ8/DR3-DQ2) as well as 375 children without this genotype were included for monthly fecal collections from 3 to 35 months of age, and genotyped for the IFIH1 polymorphisms. A total of 7,793 fecal samples were tested for presence of enterovirus RNA using real time reverse transcriptase PCR.

Results: We found no association with frequency of enterovirus in the gut for the common IFIH1 polymorphism rs1990760, or either of the rare variants of rs35744605, rs35667974, rs35337543, while the enterovirus prevalence marginally differed in samples from the 8 carriers of a rare allele of rs35732034 (26.1\%, 18/69 samples) as compared to wild-type homozygotes $(12.4 \%, 955 / 7724$ samples); odds ratio $2.5, \mathrm{p}=0.06$. The association was stronger when infections were restricted to those with high viral loads (odds ratio 3.3, 95\% CI 1.3-8.4, $\mathrm{p}=0.01$ ). The lack of association between enterovirus frequency and islet autoimmunity reported in our previous study was not materially influenced by the IFIH1 SNPs.

Conclusion: We conclude that the type 1 diabetes-associated IFIH1 polymorphisms have no, or only minor influence on the occurrence, quantity or duration of enterovirus infection in the gut. Its effect on the risk of diabetes is likely to lie elsewhere in the pathogenic process than in the modification of gut infection. 


\title{
A13
}

\section{Enterovirus and Type 1 Diabetes: review and meta-analysis of observational studies}

\author{
Lars C. Stene \\ Norwegian Institute of Public Health, Oslo, Norway
}

Introduction: No environmental risk factor has yet been established for type 1 diabetes, but enterovirus has long been suspected. A meta-analysis of studies using molecular methods of enterovirus detection was recently published by Yeung in BMJ (2011) with a pooled odds ratio around 10, but methods and results were highly heterogeneous.

Aims: To review studies of enterovirus antigen in type 1 diabetes (case control studies) or related "prediabetes" (longitudinal studies), carry out meta-analysis of studies of recent onset cases and virus genome detection in blood, and attempt to identify potential sources of bias.

Methods: "Hybrid" non-systematic review, with meta-analysis of subset of studies.

Results: While Yeung pooled about 25 heterogeneous studies with patients and controls, only eight were in my judgement suitable for a "joint" analysis. Odds ratios were relatively consistently around 10-12. Further dissection of available data (including 3 additional studies of patients without controls), showed a wide variation in the proportion of enterovirus positive patients (70-0\%), while the majority showed around $20-30 \%$ positive cases. From 0 to around 5\% of controls were positive across studies. A single laboratory had analysed samples from studies finding zero positive patients, and this laboratory has previously reported positive samples from children with "prediabetes" in several longitudinal studies. Five available longitudinal studies of "prediabetes" were heterogeneous in design and data reporting, and were thus not suitable for metaanalysis.

Conclusions: There was an intriguing tenfold higher prevalence of enterovirus antigen in the blood of newly diagnosed patients with type 1 diabetes compared to healthy controls. Assuming no major bias or confounding (which may not be reasonable...), the association could theoretically be due to causal persistent infection or some form of reverse causality. Longitudinal studies were too heterogeneous to draw any safe conclusion on association, but suggested that persistent infections were extremely rare. 


\title{
A14
}

\section{The combined effect of leisure time physical activity and diabetes on cardiovascular mortality: the HUNT Study, Norway}

\author{
Børge Moe, Eivin Eilertsen, Tom Ivar Lund Nilsen \\ Department of Human Movement Science, Faculty of Social Sciences and Technology Management, \\ Norwegian University of Science and Technology, Trondheim, Norway
}

Introduction: Diabetes increases the risk of death from cardiovascular disease, whereas physical activity is inversely associated with cardiovascular mortality. However, whether leisure time physical activity can cancel out the adverse effects of diabetes on cardiovascular mortality has not been extensively studied.

Aims: The aim of this prospective follow-up study was therefore to investigate the combined effect of leisure time physical activity and diabetes with respect to cardiovascular mortality, and to assess if physical activity could cancel out the adverse effects of diabetes.

Methods: Data on 53587 men and women without known cardiovascular disease at baseline in the population-based HUNT 2 Study (1995-97) were linked with the Cause of Death Registry at Statistics Norway.

Results: Overall, 1716 persons died from cardiovascular disease during follow-up through 2008. Compared to the reference group of physically inactive persons without diabetes, inactive persons with diabetes had an adjusted hazard ratio (HR) of 2.81 (95\% CI (confidence interval): 1.93, 4.07). The HR among persons who reported $\geq 3$ hours of light activity per week was 0.89 (95\% CI: 0.48, 1.63) if they had diabetes and $0.78(95 \%$ CI: $0.63,0.96)$ if they did not. For total physical activity, incorporating both light and hard activity, the HR among highly active persons with diabetes was 0.91 (95\% CI: 0.51, 1.60) and without diabetes the HR was 0.66 (95\% CI: 0.53, 0.81). Analysis stratified by total activity level showed a gradually weaker effect of diabetes with increasing activity level $\left(\mathrm{P}_{\text {interaction }}=0.03\right)$.

Conclusions: The results suggest that physical activity, to a large extent, may cancel out the adverse effect of diabetes on cardiovascular mortality, and that non-vigorous activity may be sufficient to obtain this favourable effect. 


\title{
A15
}

\section{Generational studies of cardiovascular risk factors in Nord-Trøndelag: The HUNT Study}

\author{
Kirsti Lund Vik, Tom Ivar Lund Nilsen \\ Department of Human Movement Science, Faculty of Social Sciences and Technology Management, \\ Norwegian University of Science and Technology
}

Introduction: The prevalence of cardiovascular risk factors, such as obesity, hypertension, hyperlipidemia, and inactivity have been rapidly increasing during the latest decades. An intergenerational study design may identify time-trends in these risk factors, and detect possible transmissions of health related behaviors. Examining how different variables interact across generations can also contribute to the use of instrumental variables to obtain unbiased estimates in observation studies.

Aims: This study investigates cardiovascular risk factors across generations. Using data from the three waves of the Nord-Trøndelag Health Study (HUNT 1, 1984-86; HUNT 2, 1995-97; and HUNT 3, 2006-08) we study if parental and offspring anthropometric factors, blood pressure, blood lipid levels, and physical activity are related.

Methods: We utilized a linkage between the separate waves of the HUNT Study to establish familial data across two generations (parents and offspring). Measures of effect are presented as coefficients from linear regression and odds ratio (OR) with 95\% confidence interval (CI) from logistic regression, adjusted for potential confounders.

Results: We found linear trends of body mass index (BMI) in offspring across categories of parental BMI. Offspring had ORs for obesity between 2.51 (2.10-2.98) and 3.28 (2.783.87 ) when one or both parents were obese (results varying by offspring and parent sex). Corresponding linear associations were found for blood pressure and blood lipid levels. ORs for hypertension were between 1.65 (1.35-2.03) and 2.26 (1.77-2.87), and for high cholesterol the ORs were between 2.27 (1.96-2.62) and 2.68 (2.33-3.08), when one or both parents were in the highest category of the variable under study. Finally, the offspring OR for being physically inactive decreased with increasing parental physical activity, with the lowest OR being 0.69 (0.52-0.91).

Conclusions: All cardiovascular risk factors in this study showed a linear association between parents and offspring. Hence cardiovascular risk factors seem to track within families. 


\section{A16}

\section{Who drinks a lot of milk?}

\section{Are Hugo Pripp}

Unit of Biostatistics and Epidemiology, Oslo University Hospital, Oslo

Introduction: Milk consumption is important in the Nordic diet and therefore relevant from a health perspective. Observed relationships between milk consumption and health could be due to nutritional properties and/or (strongly) confounded by other factors. The association between lifestyle and socio-demographic characteristics with milk consumption in a large cross-sectional population-based study was therefore assessed.

Aims: Study the relationship between lifestyle and socio-demographic characteristics with milk consumption in The Oslo Health Study (HUBRO).

Methods: The HUBRO-study was carried out from May 2000 to September 2001 with questionnaires on many factors including diet. The average score on milk consumption was categorised into three groups based on the quintiles: low (0-20\%), medium (20$80 \%)$ and high (80-100\%). Data were analysed with cross tabulation using gamma statistics $(\gamma)$ with $95 \%$ confidence intervals (95CI) for ordinal data and ordinal regression with cumulative odds ratios (ORs) as effect measures.

Results: Participants ( $\mathrm{n}=18479)$ divided into age groups 30, 40, 45, 60 and 75 years had valid data on milk consumption. High age $(\gamma=0.10$, [95CI 0.08, 0.11], p<0.001), male $(\gamma=0.12$, [95CI $0.10,0.15], \mathrm{p}<0.001)$, physical active spare time movement $(\gamma=0.13$, [95CI 0.11, 0.16], p<0.001), not smoking $(\gamma=0.08$, [95CI 0.06, 0.10], $p<0.001)$ and involvement in clubs $(\gamma=0.10$, [95CI 0.08, 0.12], $\mathrm{p}<0.001)$ were by itself all associated with higher milk consumption. The 75 years old age group lacked data on work related items and was therefore excluded from multivariate ordinal regression. For participants with complete data $(\mathrm{n}=8004)$; male $(\mathrm{OR}=1.50$, [95CI 1.36, 1.64], $\mathrm{p}<0.001)$, physical exercise $(\mathrm{OR}=1.63$, [95CI 1.37, 1.93], $\mathrm{p}<0.001$ ) and never smoked $(\mathrm{OR}=1.18$, [95CI 1.06, 1.33], $\mathrm{p}=0.004$ ) were all associated with higher milk consumption after adjusting for several other variables.

Conclusions: Milk consumption was associated with a physical active lifestyle and to lesser extent with other socio-economic factors as income and work. 


\title{
B14
}

\section{The balance linoleic acid and alpha-linolenic acid in the blood affects inflammatory blood gene expression profiles}

\author{
Olsen $\mathrm{KS}^{1^{*}}$, Fenton $\mathrm{C}^{2}$, Frøyland $\mathrm{L}^{3}$, Lund $\mathrm{E}^{1}$ \\ 1. Institute of Community Medicine, University of Troms $\emptyset$, Norway \\ 2. Microarray Resource Centre Troms $\emptyset$, Faculty of Medicine, University of Troms $\emptyset$, Norway \\ 3. National Institute of Nutrition and Seafood Research (NIFES), Bergen, Norway
}

Introduction: The ratio of dietary omega- 6 and omega-3 polyunsaturated fatty acids (PUFAs) may affect onset and pathogenesis of cardiovascular disease, inflammatory and autoimmune diseases, and cancer. The PUFAs linoleic acid (LA, omega-6) and alpha-linolenic acid (ALA, omega-3) are essential to humans and must be obtained from the diet, but excessive amounts of omega- 6 fatty acids are found in the Western diet today. Several studies indicate the importance of the ratio of LA/ALA, but their potential effects during disease onset have not been extensively studied.

Aims: We investigate the ratio of LA/ALA measured in blood plasma and its effects on blood gene expression, with the aim to explore molecular effects of PUFAs in a cross section of the Norwegian female, postmenopausal population.

Methods: Samples from 259 postmenopausal women from the Norwegian Women and Cancer (NOWAC, Kvinner og Kreft) Postgenome Cohort were included. Subjects had fatty acid concentrations measured in citrate-buffered plasma using rapid gas chromatography, and gene expression was measured in whole-blood using microarrays. We compared gene expression from the highest 26 versus the lowest 26 samples according to the LA/ALA ratio, and Gene Set Enrichment Analysis (GSEA) was employed to aid functional interpretation.

Results: In samples with a high ratio of LA/ALA 742 genes were differentially expressed and 13 gene sets were significantly enriched, mainly related to inflammatory processes and DNA packaging.

Conclusion: Our findings indicate that a high ratio of LA/ALA is related to inflammatory blood gene expression profiles in a cross section of the Norwegian, female, postmenopausal population. We conclude that our results support the hypothesis that omega-3 PUFAs may have importance for disease prevention. 


\title{
B15
}

\section{Vitamin D and Staphylococcus aureus carriage - The Tromsø Staph and Skin Study}

\author{
Karina Olsen ${ }^{1,2}$, Birgit M. Falch ${ }^{3}$, Kjersti Danielsen ${ }^{2,3}$, Mona Johannessen ${ }^{4}$, \\ Johanna U. Ericson Sollid 4 , Inger Thune ${ }^{2,5}$, Guri Grimnes ${ }^{6,7}$, Rolf Jorde ${ }^{6,7}$, \\ Gunnar S. Simonsen ${ }^{1,4}$ and Anne-Sofie Furberg1,2 \\ 1 Department of Microbiology and Infection Control, University Hospital of North Norway, N-9038 Tromsø, \\ Norway \\ ${ }^{2}$ Department of Community Medicine, Faculty of Health Sciences, University of Tromsø, N-9037 Tromsø, \\ Norway \\ 3 Department of Dermatology, University Hospital of North Norway, N-9038 Tromsø, Norway \\ ${ }^{4}$ Department of Medical Biology, Faculty of Health Sciences, University of Troms $\emptyset$, N-9037 Tromsø, Norway \\ ${ }^{5}$ Department of Oncology, Oslo University Hospital, N-0424 Ullevål, Oslo, Norway \\ ${ }^{6}$ Medical Clinic, University Hospital of North Norway, N-9038 Troms $\emptyset$, Norway \\ ${ }^{7}$ Department of Clinical Medicine, Faculty of Health Sciences, University of Troms $\emptyset$, N-9037 Troms $\emptyset$, \\ Norway
}

Introduction: Nasal carriage of Staphylococcus aureus (S. aureus) is a major risk factor for infections with the bacterium. Vitamin D induces the expression of antimicrobial peptides with activity against $S$. aureus.

Aims: We studied the association between serum 25-hydroxyvitamin D (25(OH)D) and $S$. aureus nasal carriage.

Methods: Blood samples, clinical data and repeated nasal swab cultures were collected from 1,555 women and 1,225 men aged 30-87 years in the Tromsø Staph and Skin Study 2007-08, as part of the sixth Troms $\emptyset$ Study. S. aureus nasal carrier state was based on the culturing results of two samples; carrier = two positive samples and non-carrier $=$ one or none positive sample. Multivariate logistic regression analysis stratified by recognized risk factors (sex, age and smoking) and adjusted for potential confounders was used.

Results: $S$. aureus nasal carriage rates in non-smoking men and women were $34.1 \%$ and $21.3 \%$, and in smoking men and women $24.5 \%$ and $15.2 \%$, respectively. In non-smoking men ( $\mathrm{n}=992)$, we observed a $6.7 \%$ decreased risk of $S$. aureus carriage by each $5 \mathrm{nmol} / \mathrm{l}$ increase in serum 25(OH)D $(P=.001)$, and a 33\% decrease in risk of $S$. aureus carriage in the highest tertile of serum 25(OH)D compared with the lowest tertile (OR $0.67 ; 95 \% \mathrm{CI}$, $0.48-0.95)$. Proposed adequate $(\geq 75 \mathrm{nmol} / \mathrm{l})$ versus deficient $(<50 \mathrm{nmol} / \mathrm{l})$ vitamin $\mathrm{D}$ status was associated with a $48 \%$ decrease in risk of $S$. aureus carriage $(\mathrm{OR}, 0.52 ; 95 \% \mathrm{CI}$, 0.31-0.90). In non-smoking men aged 44-60 years, odds ratio for $S$. aureus nasal carriage was 0.51 (95\% CI, $0.30-0.88)$ in the highest tertile of serum 25(OH)D. In women and smokers there were no such associations.

Conclusion: Our study supports that serum vitamin D may be a determinant of S. aureus carriage of importance for the prevention of $S$. aureus disease. 


\title{
B16
}

\section{Maternal serum levels of 25-hydroxy-vitamin D during pregnancy and risk of type 1 diabetes in the offspring}

\author{
Ingvild M. Sørensen ${ }^{1}$, Geir Joner ${ }^{1,2}$, Pål A. Jenum ${ }^{3}$, Anne Eskild ${ }^{4,5}$, Peter A. Torjesen ${ }^{6}$, \\ Lars C. Stene ${ }^{7}$ \\ 1 Dept. of Pediatrics, Oslo University Hospital Ullevål \\ 2 Dept. of Health Management and Health Economics, Institute of Health and Society, University of Oslo \\ ${ }^{3}$ Dept. of Medical Microbiology, Vestre Viken Hospital Trust \\ ${ }^{4}$ Dept. of Obstetrics and Gynecology, Akershus University Hospital \\ ${ }^{5}$ Institute of Clinical Medicine, University of Oslo \\ ${ }^{6}$ Hormone Laboratory, Oslo University Hospital Aker \\ ${ }^{7}$ Norwegian Institute of Public Health, Oslo, Norway
}

Introduction: Previous studies indicate reduced risk of type 1 diabetes following intake of vitamin D supplements during pregnancy and early childhood. However, vitamin D status is also strongly influenced by skin exposure to ultraviolet light, and the serum level of 25-hydroxy-vitamin D is a good marker of the integrated effects of dietary and endogenous sources of vitamin $\mathrm{D}$.

Aims: We investigated whether lower maternal serum concentrations of 25-hydroxyvitamin D (25-OH D) during pregnancy were associated with increased risk of childhood onset type 1 diabetes.

Methods: In a prospective cohort of 29,072 pregnant women who gave birth in Norway during 1992-94, we analyzed serum samples from 109 pregnant women delivering a child who developed type 1 diabetes before 15 years of age, and 219 randomly selected control women without type 1 diabetes children. Cases were identified by linking the cohorts of women and their offspring to The Norwegian Childhood Diabetes Registry. The serum levels of 25-OH D were analyzed by radio-immunoassay.

Results: The mean serum levels of $25-0 H$ D were $65.8 \mathrm{nmol} / \mathrm{l}$, and $73.1 \mathrm{nmol} / \mathrm{l}$ in cases and controls respectively. Comparing the lowest vs. highest quartile of maternal 25-OH D in late pregnancy, odds ratio (OR) was 2.25 (95\% CI 1.14-4.46), and the test for trend (continuous) was significant ( $\mathrm{p}=0.022)$. Even after adjustment for important confounders such as season of blood sample and others, the results remained similar and significant.

Conclusions: We showed that lower maternal serum levels of 25-0H D during late pregnancy were associated with an increased risk of type 1 diabetes in the offspring. 


\title{
B17
}

\section{Epidemiology of pedestrian traffic accidents in Arkhangelsk, Russia, 2005-2010}

\author{
Alexander Kudryavtsev ${ }^{1,2}$, Johan Lund ${ }^{3}$, Andrej Grjibovski ${ }^{1,2,4}$, Alexej Maximov ${ }^{5}$, \\ Børge Ytterstad ${ }^{1}$ \\ 1. Institute of Community Medicine, University of Tromsø, Tromsø, Norway \\ 2. International School of Public Health, Northern State Medical University, Arkhangelsk, Russia \\ 3. Norwegian Directorate of Health, Oslo, Norway \\ 4. Department of Infectious Disease Epidemiology, Norwegian Institute of Public Health, Oslo, Norway \\ 5. State Road Safety Inspectorate for Arkhangelsk Region, Arkhangelsk, Russia
}

Introduction: Road traffic accidents (RTAs) are among the leading causes of death and injury in Russia. This study describes epidemiology of pedestrian RTAs in Arkhangelsk municipality (population $~ 350,000$ ) in 2005-2010. The setting represents a NorthWestern Russian urban area.

Methods: This was a descriptive study based on the registry of RTAs of the Arkhangelsk Municipal Road Safety Inspectorate (RSI). It used the data on all 4955 RTAs with injuries or fatalities registered by the RSI in 2005-2010. Registry data were analyzed by use of frequency distributions, cross tabulation, and Cochrane-Armitage test for linear trends.

Results: Altogether, there were 2565 pedestrian RTAs in Arkhangelsk in 2005-2010. Pedestrian RTAs constituted $51.8 \%$ of all RTAs. There were 118 pedestrian fatalities and 2652 pedestrian injuries. Pedestrians accounted for $54.4 \%$ of all traffic fatalities and $44.5 \%$ of all traffic injury cases. The incidence of pedestrian fatalities decreased from 6.7 per 100.000 in 2005 to 4.0 per 100.000 in 2010 but the downward trend was not significant $(\chi 2=1.09, \mathrm{df}=1, \mathrm{P}=0.168)$. The incidence of pedestrian injuries decreased from 133.4 per 100.000 in 2005 to 106.1 per 100.000 in 2010, and had significant downward trend $(\mathrm{x} 2=12.28, \mathrm{df}=1, \mathrm{P}<0.001)$. According to the police reports, $51.8 \%$ of all pedestrian RTAs were due to faults of drivers while $48.2 \%$ were related to faults of pedestrians. The most common traffic violations in the pedestrian RTAs were pedestrians' roadway crossing at improper place (34.7\% of all pedestrian RTAs) and ignoring pedestrian crossing by drivers ( $28.4 \%$ of all pedestrian RTAs).

Conclusions: There was a decrease in the incidence of pedestrian injuries in Arkhangelsk during 2005-2010 while no changes in fatalities were observed. Prevention of pedestrian RTAs remains the major challenge for Arkhangelsk road safety authorities. Preventive measures should equally address drivers and pedestrians. 


\title{
C13
}

\section{A statistical simulation model of the natural history of breast cancer}

\author{
Westvik $\mathbf{A}^{\mathbf{1}}$, Mæhlen $\mathrm{J}^{1}$, Liestøl $\mathrm{K}^{2}$ \\ ${ }^{1}$ Department of Pathology, Oslo University Hospital-Ullevål \\ 2 Department of Informatics, Oslo University
}

Introduction: Statistical modeling has been used to improve the understanding of cancer control interventions in prevention, screening, and treatment and their effects on population trends in incidence and mortality. By combining realistic assumptions about tumor initiation and tumor growth it is possible to address questions about the natural history of the malignant disease that can not be studied in clinical trials due to time, cost, medical ethics and other concerns. Such models can therefore be used to guide public health research and priorities (see cisnet.cancer.gov).

Methods: We have modeled the natural history of breast cancer in the Norwegian population during the introduction of organized mammography screening from 1996 to present. Extensive computer simulations of interactions between the parameters for tumor initiation, growth rate and detection as well as effect of hormones, enable us to determine at which parameter combinations the model accurately predicts the screeningassociated $50 \%$ increase in tumor incidence and a 5-10 mm reduction in average tumor diameter.

Results: Our results show that if we use tumors growth rates similar to those observed for clinical cancers, the number of tumors that are screening-detectable and sub-clinical is too low to reproduce the observed incidence at prevalence screening. We are able to reproduce the observed incidence at prevalence screening only if a population of subclinical tumors with slow growth rates is added to the simulations. However, the observations of high incidence and stable tumor size at repeated screenings can not be modeled by adding slow growing tumors, but these observations can be closely replicated if we allow the added tumors to exhibit non-monotonous growth kinetics.

Conclusion: The preliminary results from our statistical model suggest that some screened tumors may have non-monotonous growth kinetics i.e. after an initial growth phase their normal fate is to regress. 


\title{
C14
}

\section{Frailty modeling of age-incidence of osteosarcoma and Ewing sarcoma for individuals below 40 years}

\author{
Morten Valberg1, Tom Grotmol ${ }^{2}$, Steinar Tretli ${ }^{2}$, Marit B. Veierød ${ }^{1}$ and Odd O. Aalen ${ }^{1}$ \\ ${ }^{1}$ Department of Biostatistics, Institute of Basic Medical Sciences, University of Oslo, Norway \\ ${ }^{2}$ Cancer Registry of Norway, Institute of Population-Based Cancer Research, Oslo, Norway
}

Introduction: Osteosarcoma and Ewing sarcoma are primary bone cancers with characteristic age-incidence curves that peak in adolescence. One interpretation is that the population contains small subgroups particularly susceptible (frail) to each of these cancers, while the majority is in practice 'immune'. The frail individuals will develop the cancer relatively early, causing the population incidence to rise. When the frail individuals have developed the disease, there are basically no one left in the population with the disposition to develop the disease. The incidence rate will thus fall. This situation can be statistically modeled by a frailty model, allowing for an individual risk that increases with age and an incidence that will peak on the population level. Additionally, the rise in incidence for these cancers follows immediately after the onset of the adolescent growth spurt. This should be accounted for in the statistical model.

Aim: To model, and explain, the peaks in the incidence rates for the two cancers.

Methods: We fit two frailty models to U.S. Surveillance, Epidemiology and End Result (SEER) incidence data for Caucasians born between 1955 and 1995, and who were younger than 40 years old in 1975-2005. Both models assume a compound Poisson distributed frailty, but we develop one model that emphasizes the impact of the growth spurt on the disease development. Also, we study models where the event of having acquired the cancers by the age of 40 is conditioned upon.

Results: The model that emphasizes the impact of the growth spurt is a significant improvement from the more standard frailty model.

Conclusions: We found that individuals with a rapid growth spurt acquire the cancers sooner than they otherwise would have, if their growth had been slower. Our results support the existing evidence of an underlying susceptibility for these two cancers. 


\title{
C15
}

\section{Colorectal cancer incidence in "Smoking, Women and Cancer Study" compared with the Norwegian general female population}

\author{
Ranjan Parajuli1 ${ }^{1}$ Eivind Bjerkaas¹, Elisabete Weiderpass ${ }^{1,2,3}$, Inger T Gram¹ \\ 1. Department of Community Medicine, Faculty of Health Sciences, University of Troms $\emptyset$, Norway \\ 2. The Cancer Registry of Norway, Oslo, Norway \\ 3. Karolinska Institutet, Department of Medical Epidemiology and Biostatistics, Stockholm, Sweden
}

Introduction: Incidence rates for colorectal cancer (CRC) among Norwegian women have increased during the last decades. We examined if the incidence of CRC in the "Smoking, Women and Cancer Study" were comparable with CRC in the general female Norwegian population.

Aims: To compare the incidence of CRC among women in a pooled dataset from 35 different health surveys in Norway with the incidence of CRC in the general female population of Norway.

Methods: Data from the three counties surveys (1974; 45,980 women; aged 20-49), 40 years surveys $(1985 ; 210,889$ women aged 40-67) and Cohort of Norway $(1994 ; 73,472$ women aged above 20 years) were pooled in a data set including approximately 330,000 women. Incident primary CRC cases diagnosed until December 31, 2007 were ascertained through linkages through the Cancer Registry of Norway and the Central Population Register, and compared with CRC incidence rates from the general population.

Results: About 3,400 primary incident CRC cases were found in our cohort comprising women who were 20 years old and above at cohort enrolment. At end of the follow up most women were between 50-54 years of age. Crude incidence rate of primary colon cancer was 32 per 100,000 and primary rectal cancer was 15 per 100000 . Almost $11 \%$ of total cancer cases among women were colorectal cancer. Data from the Cancer Registry of Norway shows age specific incidence rates per 100,000 among women in the same time period and age groups to be 30.8 and 22.7 for colon and rectal cancer respectively.

Conclusion: The incidence of CRC in the pooled data set seems to correspond to the expected incidence in the general female Norwegian population of the same ages in the same time periods. Thus, studies performed within the pooled cohort study may be generalized to the entire female Norwegian population. 


\title{
C16
}

\section{Understanding recent breast cancer trends}

\author{
Harald Weedon-Fekjær' ${ }^{1}$, Kjersti Bakken ${ }^{2,3}$, Lars Vatten ${ }^{4}$ and Steinar Tretli ${ }^{5,6}$ \\ 1. Department of Etiological Research, Cancer Registry of Norway, Norway \\ 2. University of Troms $\emptyset$, Norway \\ 3. University of Bergen, Norway \\ 4. Department of Public Health, Norwegian University of Science and Technology, Trondheim, Norway \\ 5. Cancer Registry of Norway, Norway \\ 6. Norwegian University of Science and Technology, Norway
}

Introduction: After decades of consistent increase, the incidence of breast cancer has recently declined in many developed countries. Reduced use of hormone therapy [HT] and completion of the first wave of mammography screening have been suggested as explanatory factors, but each factor's contribution is still uncertain. The nationwide cancer registry, the gradually extended public mammography screening program, and county specific recordings of HT sales, make Norway well suited to study how mammography screening and HT use may influence breast cancer incidence trends.

Methods: An extended age-period-cohort [APC] model, with information on HT sales and stage of the mammography screening program, was applied on aggregated Norwegian county specific data, including 50102 invasive breast cancer cases diagnosed among Norwegian women 30-90 years of age between 1987 and 2008.

Results: The incidence of breast cancer in Norway increased steadily until 2002, before it leveled off, and a decline became apparent from 2006. All non-linear changes in the incidence were explained by HT use and mammography screening, with approximately similar contributions of each factor. In 2002, when the incidence rate among women 5069 years of age was highest, we estimated that 23 percent of the cases in that age group could be attributed to screening mammography, and 27 percent to use of hormone treatment. The relative risk of breast cancer associated with current use of HT was 2.2 $\{95 \% \mathrm{CI}: 1.9,2.5\}$, with a short lag time from exposure to increased risk.

Conclusion: Changes in breast cancer incidence trends since the early 1990s may be fully attributed to mammography screening and hormone treatment, with about similar contributions of each factor. The effect of HT on breast cancer incidence in Norway appears to be larger than expected based on the results of US randomized trials. 
Den 19. norske epidemiologikonferansen, Holmen fjordhotell 17.-18. november 2011

Deltakerliste

\begin{tabular}{|c|c|c|}
\hline Etternavn & Fornavn & E-postadresse \\
\hline Abdelnoor & Michael & uxmiab@ous-hf.no \\
\hline Abebe & Dawit Shawel & dawit.abebe@nova.no \\
\hline Andersen & Bror Just & BROAND@vestreviken.no \\
\hline Biehl & Anna Månsson & anna.biehl@fhi.no \\
\hline Bjerkaas & Eivind & eivind.bjerkaas@uit.no \\
\hline Brantsæter & Anne Lise & AnneLise.Brantsaeter@fhi.no \\
\hline Brunborg & Cathrine & uxbruc@ous-hv.no \\
\hline Czajkowski & Nikolai & Nikolai.Czajkowski@fhi.no \\
\hline Dahl & Cathrine & Cathrine.Dahl@fhi.no \\
\hline Dahl & Cecilie & Cecilie.Dahl@fhi.no \\
\hline Eggesb $\varnothing$ & Merete & Merete.Eggesbo@fhi.no \\
\hline Fagerland & Morten Wang & morten.fagerland@medisin.uio.no \\
\hline Flaten & Trond & trond.flaten@chem.ntnu.no \\
\hline Fleten & Anne Caroline & AnneCaroline.Fleten@fhi.no \\
\hline Forsén & Lisa & Lisa.Forsen@fhi.no \\
\hline Furu & Kari & Kari.Furu@fhi.no \\
\hline Graff-Iversen & Sidsel & Sidsel.Graff.Iversen@fhi.no \\
\hline Gran & Jon Michael & j.m.gran@medisin.uio.no \\
\hline Grjibovski & Andrej & andrei.grjibovski@fhi.no \\
\hline Grotmol & Tom & Tom.Grotmol@kreftregisteret.no \\
\hline Grøtvedt & Liv & Liv.Grotvedt@fhi.no \\
\hline Handal & Marte & Marte.Handal@fhi.no \\
\hline Hestvik & Unn & unn.hestvik@kreftregisteret.no \\
\hline Hjellvik & Vidar & Vidar.Hjellvik@fhi.no \\
\hline Hold $\varnothing$ & Ingvild & ingvild.holdo@studmed.uio.no \\
\hline Holvik & Kristin & Kristin.Holvik@fhi.no \\
\hline Hovengen & Ragnhild & Ragnhild.Hovengen@fhi.no \\
\hline Håberg & Siri Eldevik & SiriEldevik.Haberg@fhi.no \\
\hline Iversen & Marjolein & Marjolein.Iversen@hib.no \\
\hline Jakopanec & Irena & irena.jakopanec@dnv.no \\
\hline Jani-Bølstad & Jagrati & jagrati.jani-bolstad@medisin.uio.no \\
\hline Jansen & Mona Drevdal & Mona.Dverdal.Jansen@fhi.no \\
\hline Joner & Geir & geir.joner@medisin.uio.no \\
\hline Knudsen & Gun Peggy & GunPeggy.Knudsen@fhi.no \\
\hline Krieger & Ekaterina & kate.krieger@mail.ru \\
\hline Krog & Norun Hjertager & NorunHjertager.Krog@fhi.no \\
\hline Kudrayavtsev & Alexander & ispha09@gmail.com \\
\hline Langseth & Hilde & Hilde.Langseth@kreftregisteret.no \\
\hline Larsen & Inger Kristin & inger.kristin.larsen@kreftregisteret.no \\
\hline Lie & Kari Kveim & KariKvem.Lie@fhi.no \\
\hline Lier & Ragnhild & ragnhild.lier@svt.ntnu.no \\
\hline Lindman & Anja Schou & Anja.Schou.Lindman@kunnskapssenteret.no \\
\hline Liu & Hai-Ying & Hai-Ying Liu@nilu.no \\
\hline Madsen & Christian & Christian.Madsen@fhi.no \\
\hline Magnus & Maria Christine & Maria.Christine.Magnus@fhi.no \\
\hline Misiri & Humphrey & hmisiri@gmail.com \\
\hline Moe & Børge & borge.mo@svt.ntnu.no \\
\hline Moger & Tron Anders & tronmo@medisin.uio.no \\
\hline Nafstad & Per & per.nafstad@medisin.uio.no \\
\hline
\end{tabular}




\begin{tabular}{|c|c|c|}
\hline Nilsen & Tom IL & Tom.Ivar.Lund.Nilsen@svt.ntnu.no \\
\hline Nordeng & Hedvig & h.m.e.nordeng@farmasi.uio.no \\
\hline Nystad & Wenche & Wenche.Nystad@fhi.no \\
\hline Næss & Øyvind & Oyvind.Naess@fhi.no \\
\hline Oftedal & Bente & bente.oftedal@fhi.no \\
\hline Olsen & Karina & Karina.Olsen@unn.no \\
\hline Olsen & Lena Ringstad & Lena.Ringstad.Olsen@unn.no \\
\hline Olsen & Karina Olsen & karinasolsen@gmail.com \\
\hline Omsland & Tone Kristin & tone.kristin.Omsland@fhi.no \\
\hline Opdahl & Signe & signe.opdahl@ntnu.no \\
\hline Panasevich & Sviatlana & Sviatlana.Panasevich@fhi.no \\
\hline Parr & Christine & c.l.parr@medisin.uio.no \\
\hline Pripp & Are Hugo & apripp@ous-hf.no \\
\hline Rabanal & Kjersti Stormark & kjerab@ous-hf.no \\
\hline Ranjan & Parajuli & ranjan.parajuli@uit.no \\
\hline Reichborn-Kjennerud & Ted & Ted.Reichborn-Kjennerud@fhi.no \\
\hline Røysland & Kjetil & kjetil.roysland@medisin.uio.no \\
\hline Samuelsen & Sven Ove & osamuels@math.uio.no \\
\hline Sandven & Irene & uxsair@ous-hf.no \\
\hline Selmer & Randi & Randi.Selmer@fhi.no \\
\hline Skudutyte-Rysstad & Rasa & rasa.skudutyte-rysstad@odont.uio.no \\
\hline Skurtveit & Svetlana & Svetlana.Skurtveit@fhi.no \\
\hline Solvang & Hiroko & Hiroko.Solvang@rr-research.no \\
\hline Steingrímsdóttir & Ólöf Anna & OlofAnna.Steingrimsdottir@fhi.no \\
\hline Stene & Lars Christian & Lars.Christian.Stene@fhi.no \\
\hline Stigum & Hein & Hein.Stigum@fhi.no \\
\hline Storeng & Ritsa & rstoreng@ous-hf.no \\
\hline Strand & Bjørn Heine & BjornHeine.Strand@fhi.no \\
\hline Støer & Nathalie & nathalcs@math.uio.no \\
\hline Syse & Astri & Astri.Syse@kreftregisteret.no \\
\hline Søgaard & Anne Johanne & Anne.Johanne.Sogaard@fhi.no \\
\hline Sørensen & Ingvild Menes & i.m.sorensen@medisin.uio.no \\
\hline Sørensen & $\varnothing y s t e i n$ & oystein.sorensen@medisin.uio.no \\
\hline Thoresen & Magne & magne.thoresen@medisin.uio.no \\
\hline Torgersen & Leila & Leila.Torgersen@fhi.no \\
\hline Tretli & Steinar & Steinar.Tretli@kreftregisteret.no \\
\hline Usynina & Anna & perinat@mail.ru \\
\hline Valberg & Morten & morten.valberg@medisin.uio.no \\
\hline Vik & Kirsti Lund & kirsti.lund.vik@svt.ntnu.no \\
\hline Vikanes & Åse Vigdis & Ase.Vigdis.Vikanes@fhi.no \\
\hline Vårdal & Mari & mavaar@ous-hf.no \\
\hline Walnum & Alexander & alexander.walnum@skde.no; \\
\hline Wang & Nina & nina@wang@odont.uio.no \\
\hline Weedon-Fekjær & Harald & Harald.Weedon-Fekjaer@kreftregisteret.no \\
\hline Westvik & Asbjørn & asbjorn.westvik@gmail.com \\
\hline Wits $\varnothing$ & Elisabet & Elisabet.Witso@fhi.no \\
\hline Ystrøm & Eivind & Eivind.Ystrom@fhi.no \\
\hline Zahl & Per Henrik & Per-Henrik.Zahl@fhi.no \\
\hline Aalen & Odd & o.o.aalen@medisin.uio.no \\
\hline Aamodt & Geir & Geir.Aamodt@fhi.no \\
\hline Ånonsen & Olga & Olga.anonsen@fhi.no \\
\hline Aasvang & Gunn Margit & Gunn.Margit.Aasvang@fhi.no \\
\hline
\end{tabular}

